\title{
Endogenous scheduling preferences and congestion $^{1}$
}

\author{
Mogens Fosgerau ${ }^{2}$ \\ Technical University of Denmark, Denmark \\ Royal Institute of Technology, Sweden \\ Kenneth Small ${ }^{3}$ \\ University of California, Irvine \\ Resources for the Future, Washington, D.C.
}

May 25, 2014

\footnotetext{
${ }^{1}$ We are grateful for comments by Richard Arnott, Clifford Winston, participants in several conferences, and anonymous referees. All responsibility remains with the authors. Mogens Fosgerau has received support from the Danish Strategic Research Council.

${ }^{2} \mathrm{mf} @$ transport.dtu.dk

${ }^{3}$ ksmall@uci.edu
} 


\begin{abstract}
We seek to better understand the scheduling of activities in time through a dynamic model of commuting with congestion, in which workers care solely about leisure and consumption. Implicit preferences for the timing of the commute form endogenously due to concave preferences and temporal agglomeration economies. Equilibrium exists uniquely and is indistinguishable from that of a generalized version of the classical Vickrey bottleneck model, based on exogenous trip-timing preferences; but optimal policies differ: the Vickrey model will under-predict the benefits of congestion pricing, and such pricing may make people better off even without considering the use of revenues.
\end{abstract}

Keywords: urban congestion; agglomeration; endogenous preferences; scheduling preferences; bottleneck

JEL codes: D11, R41

\title{
1 Introduction
}

The scheduling of people's activities determines many economic actions and investment needs. In particular, the tendency towards wanting to do similar things at the same time results in many significant expenses, for example large sports stadia, concert halls, and convention centers. This synchronization, along with a desire for spacious residential surroundings, also requires expensive peak-capacity communications links and traffic arteries in order to enable people to communicate or congregate simultaneously.

Yet standard economic tools do not deal well with these phenomena because they typically involve some sort of increasing returns to scale for activities at a given location, as elegantly explained by Starrett (1974) and Krugman (1991). These and other authors have developed the consequences of such spatial "agglomeration economies", showing how they produce product differentiation (involving scale economies for a given product variety), pecuniary externalities (by which one firm's competitive action or one consumer's preferences affect others' ability to reap scale economies), and spatial concentration. 
Similarly, the advantages of concentrating many individuals' and firms' activities in time seem likely to create new phenomena. In particular, congestion in transportation results because the demands for moving people and goods are agglomerated in space and time. Thus understanding it requires being able to model explicitly how those demands and the congestion resulting from them are simultaneously determined.

Of the two types of agglomeration, the spatial type is much better understood (see e.g. Rosenthal and Strange, 2004). Researchers have learned much about the strength of spatially agglomerating forces such as labor market pooling, knowledge transmission, and building of trust — each at a variety of geographical levels including regions, metropolitan areas, urban subcenters, and even small industrial districts. ${ }^{1}$ In some cases, explicit models can be solved to explain complex equilibrium spatial patterns, such as those studied by Fujita and Ogawa (1982) and Lucas and Rossi-Hansberg (2002) concerning the internal structure of metropolitan areas. Most of this work is concerned with productivity at the workplace. Some, such as Glaeser et al. (2001), also consider the value of a location to consumers.

Temporal agglomeration, by contrast, has elicited a much sparser literature. Henderson (1981) shows that if workers are more productive when large numbers are at work simultaneously, and wages reflect those productivity differences, then workers are induced to find an equilibrium that produces temporal clustering and hence traffic congestion. Wilson (1988) provides supporting empirical evidence. ${ }^{2}$ Vovsha and Bradley (2004) show empirically that the timing of work trips involves preferences related both to the workplace and to the home: an example of the latter is an aversion to departing from home too early in the day or returning too late. Thus, in addition to the strong support for workplace agglomeration based on productivity, there is some evidence that people also care about the timing of their activities at home.

\footnotetext{
${ }^{1}$ See for example Chinitz (1961) and Scott (1988) on central business districts; Anas and Kim (1996) and Helsley and Sullivan (1991) on urban subcenters; Glaeser and Gottlieb (2009) on metropolitan areas; and Krugman (1991) on regions.

${ }^{2}$ Arnott (2007) reviews these papers and further applications, while adding his own innovation (still within a static framework) by allowing aggregate labor supplied to be affected by congestion tolls via a reduction in the net wage. Gutierrez-i Puigarnau and Van Ommeren (2012), on the other hand, present evidence suggesting that the relationship may be weak empiricaly.
} 
However, there has been only limited success with modeling the simultaneous formation of these scheduling preferences and of congestion. The problem is difficult because it is inherently dynamic as well as nonlinear. As an example of the difficulties encountered, Henderson (1981) derives an equilibrium pattern from his model of workplace productivity, but he is forced to assume travel times are determined in a way that allows for overtaking of earlier vehicles by ones departing later. Small and Chu (2003) derive an equilibrium congestion pattern in a dense downtown street network, but are forced to make a different unrealistic assumption, namely that travel time is determined solely by traffic density at the end of the trip.

The most successful theoretical models of equilibrium temporal aggregation rely instead on exogenous scheduling preferences. Vickrey (1969) and many successors such as Arnott et al. $(1990,1993)$ assume that each worker has a predetermined preferred work arrival time and suffers disutility from deviating from that time. These papers describe congestion as a deterministic queue behind a bottleneck, and this description has enabled them to shed light on numerous questions including the effects of heterogeneous users, parallel and serial routes, and various pricing and investment strategies. For useful reviews, see Arnott et al. (1998) or Small and Verhoef (2007).

This paper returns to the problem of understanding the origin of scheduling preferences. We address agglomeration in time not only in the workplace but also at another location, here described as "home," where non-work activities ("leisure") take place. The result is a firmer microfoundation for the demand for travel, based on a few simple technological relationships along with the assumption that people choose schedules to maximize their combined utility of work and leisure. We are able to show that equilibrium exists and explore its properties.

We do so by making strong simplifying assumptions about the nature of the agglomerative forces and of the travel network connecting the locations where they occur. First, we ignore heterogeneity in order to highlight the role of endogenous preferences. Thus we rule out certain empirically observed phenomena, such as people commuting completely outside the normal peak hours and occupations requiring multiple shifts.

Second, we assume that worker productivity increases with the number of 
people simultaneously at work, and that utility from leisure similarly increases with the number of people simultaneously at the non-work location. We show that these assumptions produce temporal clustering of both work and leisure, with congested travel connecting them. The assumption about workers is conventional: for example, Henderson (1981) notes that "[w]hite collar jobs involve extensive communications and interactions with corresponding people in opposing or complementary firms. The larger the mass of people at work [at a given time] the greater will be their individual productivity." This argument applies within firms, whose very existence supposes a need for close intra-firm coordination, and also between firms to the extent that a firm's success requires interaction with other firms. For example, efficient trading of securities may require a single set of opening hours for a stock exchange. As another, families with children are constrained by the opening hours of child care institutions and schools, which are in turn determined in response to normal working hours, providing an additional mechanism through which workers are more productive (net of child care costs) when working simultaneously. As these examples illustrate, some populationwide agglomeration economies are likely to remain even after various subgroups adopt coordinating measures, such as work-hour conventions, that reduce them. ${ }^{3}$

In the spatial dimension, such scale economies have been measured empirically and are of sufficient magnitude that they are thought by most urban economists to underlie the formation of cities and especially of central business districts. ${ }^{4}$ In the temporal dimension, the willingness of firms and workers to operate at hours requiring commuting during peak-hour traffic congestion implies that temporal agglomeration is important, and Wilson (1988) confirms empirically that wages vary with work hours as such temporal agglomeration would imply.

Our arguments for supposing an agglomeration effect related to leisure are basically the same, although there is less empirical evidence regarding its magnitude. Numerous leisure activities require simultaneous participation: e.g. telephone calls, family meals, social events, sports, online games. If more people are off work at a given time, then there are fewer constraints limiting the formation

\footnotetext{
${ }^{3}$ We thank a referee for some of the examples in this paragraph.

${ }^{4}$ A classic statement is by Chinitz (1961). Duranton and Puga (2004) provide a thorough modern examination of the sources and effects of urban agglomeration economies.
} 
of such activities, even when they involve only a few people. Just as with work agglomeration, conscious coordination can facilitate such simultaneous participation, but at a cost that varies inversely with the temporal density of available people. And some such activities cannot easily be coordinated, indeed some thrive on spontaneity. For example, many high-rent urban residential districts attract people who seek an active social life filled with busy bars, night clubs, streets, and theaters, all of which require a high spatial and temporal density of clients. Empirical studies, e.g. Glaeser et al. (2001), have found that the kinds of urban amenities fostered by high density attract residents to a given metropolitan area; and Tabuchi and Yoshida (2000) find consumption agglomeration economies to be of comparable magnitude to workplace agglomeration economies. The likelihood that such agglomeration forces in leisure are temporal as well as spatial has led researchers to posit a time-varying utility of time spent at home (e.g. Vickrey (1973), Tseng and Verhoef (2008)), the most likely explanation for which is the possibilities for interacting with other people who are also not at work.

We carry out a detailed comparison of our results with those of the most similar model using exogenous preferences, namely Vickrey (1973). That paper in turn is similar in spirit to, but somewhat more general than, the more common "bottleneck model" of Vickrey (1969). It turns out that in our model, scheduling preferences of the kind assumed by Vickrey (1973) arise endogenously in equilibrium. That is, an individual taking as given the equilibrium pattern of departures from home, as well as work and leisure productivities, will appear to behave according to scheduling preferences in the form of a utility function that depends on when the commute starts and ends. The scheduling preferences of Vickrey (1973) belong to a general class that, as far as we are aware, comprises all specifications considered by Vickrey and later authors in the context of the bottleneck model. ${ }^{5}$ We derive some properties of Nash equilibrium for this general class, in order to compare its predictions to those of our model. This allows us to evaluate and in some cases sign the errors that result if policies aimed at regulating congestion are developed assuming (incorrectly) that scheduling preferences are exogenous.

In order to make our analysis tractable, we restrict it to the case where con-

\footnotetext{
${ }^{5}$ Actually the "bottleneck model" is a limiting case of the more general model, but one that is easy to handle analytically.
} 
sumers are homogeneous. Actually Vickrey's 1969 paper allowed commuters to have different preferred arrival times, as does Newell (1987). Other papers allow for heterogeneity in the parameters of users' travel-cost function (Hendrickson and Kocur (1981); Cohen (1987); Lindsey (2004); van den Berg and Verhoef (2011)). Heterogeneity clearly leads to interesting results and greater realism, at the cost of a rapid increase in model complexity; we hope our model can be extended in this way in the future.

We find that the assumption of exogenous scheduling preferences would lead an analyst to underestimate the benefit of congestion tolling. Using numerical simulations, we also find that in most cases, such an analyst would underestimate the direct benefits to travelers (not counting toll revenues); this means that people can in fact be made better off by pricing, even when not accounting for how toll revenues are used. This suggests that one key to gaining acceptance of congestion pricing might be to explain intuitively how it would help people achieve the benefits of agglomeration at work and home.

Humans are social animals and so it is entirely natural that the scheduling preferences of one individual should depend on the scheduling choices of others. Indeed, traffic congestion may be viewed as an example of the many social interactions that shape economic behavior (Manski, 2000). In our model the interaction occurs roughly at the level of a city, but there are many smaller-scale interactions that may also lead to situations where people trade off a desire to be together against resource barriers to doing so. For example, one could consider the problem of arranging appointments within small groups of people who compete with other groups for meeting space: their apparent preferences over meeting schedules might arise from factors similar to those analyzed here.

\section{Model with endogenous scheduling preferences}

We consider a continuum of $N$ homogenous workers. Utility is a differentiable and strictly increasing function of "effective leisure" $H$ produced at home and of consumption $Z$, which is equal to "output" $W$ produced at work, with the interpretation that work output is exchanged for consumption at a constant price normalized to one. When there is no toll to be subtracted from consumption, util- 
ity is thus simply $U(H, W)$.We impose the following conditions on the utility function:

Condition $1 U(H, W) \rightarrow-\infty$ as either $H \rightarrow 0$ or $W \rightarrow 0$.

Condition 2 If $H_{a}<H_{b}$ and $W_{a}>W_{b}$, then

$$
\frac{U_{H}\left(H_{a}, W_{a}\right)}{U_{W}\left(H_{a}, W_{a}\right)}>\frac{U_{H}\left(H_{b}, W_{b}\right)}{U_{W}\left(H_{b}, W_{b}\right)} .
$$

Condition 1 is needed only to rule out the extreme outcomes $H=0$ or $W=0$; a weaker and quite realistic version would suffice but at some cost of complexity. ${ }^{6}$ Condition 2 will ensure that Nash equilibrium is unique. It states that the marginal rate of substitution between leisure and consumption decreases as leisure increases and consumption decreases; together with assumptions that utility is strictly increasing and differentiable, this condition implies strict concavity of the utility function.

We now describe agglomeration. Let $[0, \Omega]$ denote the available time interval defining the morning. Worker productivity (aggregate output per worker per unit time) at any time $t$ is positively related to the number of workers at work at that time. Since we are not trying to analyze the productivity effects of absolute city size, we will take agglomeration to depend on the share of workers who are at work at time $s, N_{W}(s) / N$, through an agglomeration function $g_{W}(\cdot)$. That is, each worker who is at work at time $s$ produces output at rate $w(s) \equiv g_{W}\left[N_{W}(s) / N\right]$. We assume $g_{W}$ is differentiable (hence continuous) and increasing. A worker who arrives at work at time $a$ produces total output

$$
W(a)=\int_{a}^{\Omega} w(s) d s
$$

We assume $g_{W}(0)=0$, which implies that no production takes place when a single (mass zero) worker is alone at work. This is a strong assumption but

\footnotetext{
${ }^{6}$ Specifically, this condition can be replaced by requiring that both $U(0, W)$ and $U(H, 0)$ are less than $U(H, W)$ whenever $H, W>0$.
} 
maintained for reasons of analytical tractability; we discuss relaxing it in the Conclusion. Without loss of generality, we normalize $g_{W}(1)=1$.

Similarly, effective leisure is produced in a social context, with an increasing and differentiable agglomeration function $g_{H}(\cdot)$, where $N_{H}(s)$ is the number of people at home at time $s$. Effective leisure for a worker is produced at time $s$ at rate $h(s) \equiv g_{H}\left[N_{H}(s) / N\right]$, so that a worker who remains at home until time $t$ produces effective leisure

$$
H(t)=\int_{0}^{t} h(s) d s
$$

We assume $g_{H}(0)=0$ and normalize $g_{H}(1)=1$. Leisure can be interpreted as home production subject to agglomeration economies.

For later convenience, we define the cumulative agglomeration functions $G_{W}(z) \equiv$ $\int_{0}^{z} g_{W}(x) d x$ and $G_{H}(z) \equiv \int_{0}^{z} g_{H}(x) d x$. Our definitions and normalizing assumptions imply that $0<G_{H}(1)<1$ and $0<G_{W}(1)<1$.

We are interested in commuting between home and work. Given the symmetry with which we model effective leisure and output, it should not matter whether we consider the trip to or from work; for simplicity we consider just the first. ${ }^{7}$ Thus we require each worker to start the morning at home and end it at work. Travel between home and work occurs through a one-way bottleneck with a capacity of $\psi$ workers per time unit. Travel time required before or after the bottleneck is identical for everyone at a constant value, which we normalize to zero. This normalization comes at no loss of generality so long as there is sufficient capacity for everyone to complete their travel by the end of the morning and still have time left over for leisure and work; thus we assume $\psi \Omega>N$. The queueing technology is as described by Vickrey (1969): A bottleneck is served at the rate $\psi$ and a vertical queue (i.e., one whose physical extent can be ignored) with first-infirst-out queue discipline builds up whenever travelers arrive at the bottleneck at a rate faster than $\psi$.

\footnotetext{
${ }^{7}$ We make no assumptions about when a given worker would return home. Notice we put no structure on the model that would cause an interdependence between the times at which the trip to work and the return home are undertaken; thus, if we were to model the latter, it would be a mirror image of the trip to work.
} 
At any time $t$, the numbers of workers at home, traveling, and at work are $N_{H}(t), N_{T}(t)$, and $N_{W}(t)$, respectively, such that $N_{H}(t)+N_{T}(t)+N_{W}(t)=N$ for all $t \in[0, \Omega]$. Each worker remains at work until time $\Omega$.

\subsection{Nash equilibrium}

Nash equilibrium occurs when no worker can gain from a unilateral change of departure time. With identical workers, this translates into the condition that utility achieved by choosing a given departure time is constant over the set of those times when departures actually occur, and that utility would be smaller for a departure time outside this set.

Workers depart from home (and arrive at the bottleneck) during an endogenously determined interval $\left[t_{0}, t_{1}\right]$. We will make use of the relative departure time, $t_{d}=t-t_{0}$. Let $\rho\left(t_{d} \mid t_{0}\right)$ be the departure rate from home at time $t_{0}+t_{d}$, so that the cumulative number of departures is $R\left(t_{d} \mid t_{0}\right) \equiv \int_{0}^{t_{d}} \rho\left(s \mid t_{0}\right) d s$. The following lemma establishes the basic characteristics of the payoff functions the individual traveler faces as he chooses a departure time. (All proofs are given in appendix A.)

Lemma 1 Suppose departures take place during an interval $I=\left[t_{0}, t_{1}\right] \subset[0, \Omega]$, where $t_{1}=t_{0}+N / \psi$, and where there is a queue for all $t \in \operatorname{int}(I)$. Then the rate of arrivals at work is $\psi$ during interval I and zero elsewhere. For a worker choosing departure time $t$, effective leisure is

$$
H(t)=\left\{\begin{array}{cc}
t & \text { if } t<t_{0} \\
t_{0}+\int_{0}^{t-t_{0}} g_{H}\left(1-\frac{R\left(t_{d} \mid t_{0}\right)}{N}\right) d t_{d} & \text { if } t \geq t_{0}
\end{array}\right.
$$

which is (weakly) increasing and concave in t; and output at work is

$$
W(a)=\left\{\begin{array}{cc}
\Omega-t_{0}-\frac{N}{\psi}+\frac{N}{\psi} G_{W}(1) & \text { if } a<t_{0} \\
\Omega-t_{0}-\frac{N}{\psi}+\frac{N}{\psi} \cdot\left[G_{W}(1)-G_{W}\left(\frac{\psi \cdot\left(a-t_{0}\right)}{N}\right)\right] & \text { if } t_{0} \leq a \leq t_{1} \\
\Omega-a & \text { if } a>t_{1}
\end{array}\right.
$$

which is (weakly) decreasing and concave in a. These properties are strict for $t$ 
and $a$ in the open interval $\left(t_{0}, t_{1}\right)$.

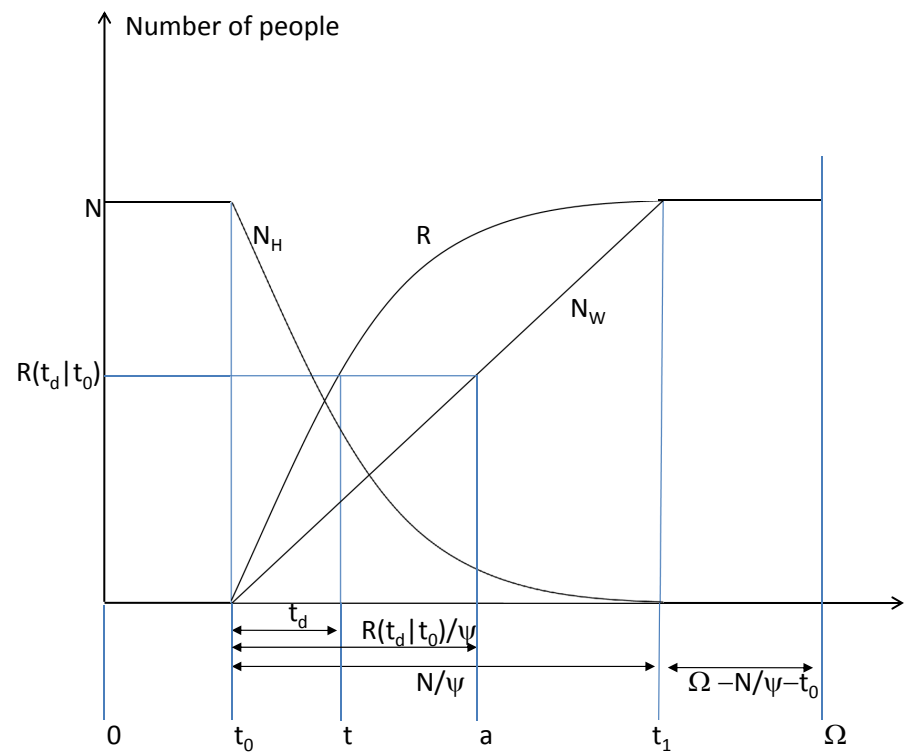

Figure 1: Cumulative departures $(R)$, and number of workers at home $\left(N_{H}\right)$ and at work $\left(N_{W}\right)$

Lemma 1 may be visualized through Figures 1 and 2. Figure 1 shows the number of workers at home and at work as a function of time, along with cumulative departures (the concave curve in the figure). Figure 2 depicts the resulting accumulation of work and leisure for a traveler who chooses to depart at some time $t \in$ $\left[t_{0}, t_{1}\right]$. Effective leisure accumulates at rate $h(s)=g_{H}\left[1-R\left(s-t_{0} \mid t_{0}\right) / N\right]$, starting at time $s=t_{0}$ and continuing until $s=t$ (or until all other travelers have departed, if earlier). Work output accumulates at rate $w(s)=g_{W}\left[\psi \cdot\left(s-t_{0}\right) / N\right]$, starting at time $s=a$ when the traveler arrives (or the time the first other person arrives, if later) and continuing until the end of the morning, $s=\Omega$. The area under $w(s)$ between times $t_{0}$ and $t_{1}$ is $\int_{t_{0}}^{t_{1}} g_{W}\left(\psi \cdot\left(s-t_{0}\right) / N\right) d s=(N / \psi) G_{W}(1)$; that between times $t_{0}$ and $a$ is $\int_{t_{0}}^{a} g_{W}\left(\psi \cdot\left(s-t_{0}\right) / N\right) d s=(N / \psi) G_{W}\left[\psi \cdot\left(a-t_{0}\right) / N\right]$. The former area gives the value of $W$ accumulated during time interval $\left[t_{0}, t_{1}\right]$ for someone who arrives at work before or at $t_{0}$, while the difference between the two 


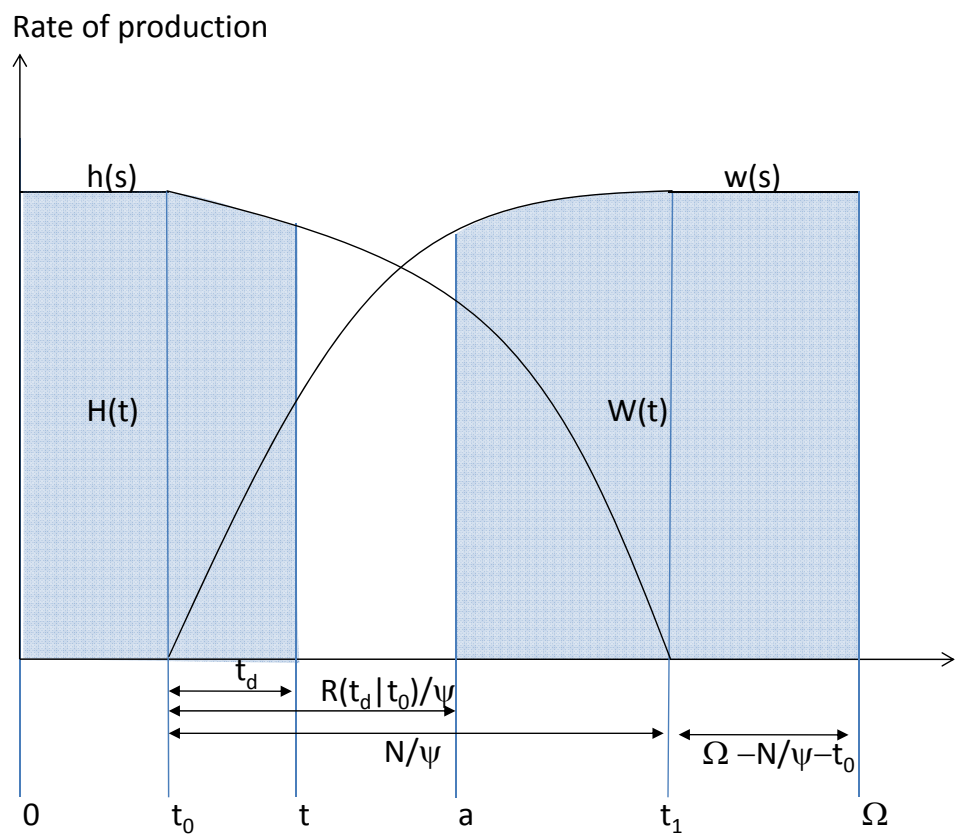

Figure 2: Instantaneous rates of production (curves $h, w$ ) and total production for worker departing at time $t$ (areas $H, W$ ) 
areas gives that value for someone arriving at a time $a>t_{0}$ such as shown in the figure. In both cases, this person also receives a contribution to $W$ equal to the rectangle to the right of $t_{1}$ : that is, $\Omega-t_{0}-N / \psi$.

The following theorem establishes key properties of equilibrium. In particular, it establishes that the departure interval has duration just long enough to allow the bottleneck to deliver all $N$ workers to their destinations, and that it begins at a unique point in time that allows utility to be equalized for the first and last travelers.

Theorem 1 (Nash equilibrium). Nash equilibrium exists uniquely. In Nash equilibrium, departures and arrivals take place during an interval $I=\left[t_{0}, t_{1}\right] \subset$ $(0, \Omega)$ satisfying

$$
t_{1}=t_{0}+N / \psi
$$

Utility is constant on this interval and in particular is equal at the end points:

$$
U\left[H\left(t_{0}\right), W\left(t_{0}\right)\right]=U\left[H\left(t_{1}\right), W\left(t_{1}\right)\right] .
$$

The departure schedule $R\left(\cdot \mid t_{0}\right)$ is strictly concave on $[0, N / \psi]$ and there is always a strictly positive queue on the interior of this interval.

The cumulative departure rate is determined by the fact that Nash equilibrium requires workers to be indifferent among all active departure times. The extreme undesirability of either zero output or zero effective leisure, assumed in Condition 1 , bounds $I$ away from the ends of the available day. There must be a queue throughout this interval except at its extreme limits, since otherwise commuters suffering a queue would switch to a nearby time with no such queue. There is no residual queue at the end of the departure interval, since otherwise the last person to depart could postpone departure slightly and gain utility. The uniqueness of the equilibrium is ensured by Condition 2 .

While our conditions fully determine the location of the departure interval $I$ within $[0, \Omega]$, our defined "day", its location in clock time is unspecified here because the clock times we have labeled 0 and $\Omega$ are arbitrary. In other words, our model does not specify whether people view home production as starting at 5:00 a.m., 6:00 a.m., or some other time. This extra degree of freedom does not 
interfere with our results, but if one wanted to explain the actual clock times of rush hours one would need to add some absolute preference over clock times. It seems likely that the most important such preference is that the "day" be roughly centered around hours of natural sunlight, since we observe in cities everywhere that the most common work hours occur during such hours. Alternatively, one could assume that worker productivity is highest during times of sunlight. Other possible factors explaining clock times include cultural preferences (perhaps not very stable) and coordination with activities in other cities. The latter would tend to cause cities on the edge of a time zone to have an imperfect correspondence between work and daylight - Chicago, Boston, and all of Spain come to mind as examples since they are all located where the sun is highest well before or after 12:00 noon as measured by their time zone.

\section{Model with exogenous scheduling preferences}

For comparison, we now describe a more conventional model where scheduling choices arise from fixed scheduling preferences - that is, preferences that are functions of clock time. Such preferences have typically been constructed around an assumed ideal work start time, with utility penalties for arriving earlier or later than that time. Occasionally, such preferences have instead been built from an assumed schedule giving instantaneous rates of accumulation of utility as functions of clock time. Here, we introduce a generalized version that incorporates either of these motivations. The key point is that preferences are defined in terms of specific times of day. In this section, we derive properties of this model; in the next section, we contrast them with our model of endogenous scheduling.

Let each person's utility be a function $V(t, a)$ of departure and arrival times, and let this function be strictly concave, increasing in $t$, and decreasing in $a$. Defining $\widetilde{V}(t)=V(t, t)$ as the utility achived by someone suffering no queueing delay, assume that $\widetilde{V}(t)$ attains a maximum at some value of $t$. In other words, there is something exogenous generating a peaked preference for particular clock times for travel. Again we assume $N$ identical users and a deterministic bottleneck with capacity $\psi$, and we consider Nash equilibrium in departure times. We call this model "the general Vickrey model". 
In one special case, $V(\cdot)$ is derived as the integral of instantaneous utility rates $v_{H}(t)$ and $v_{W}(t)$ for time spent at home and work, respectively; see Vickrey (1973), Tseng and Verhoef (2008), and Fosgerau and Engelson (2011). In another case, actually a limiting case since $V$ is now concave but not strictly so, $V(t, a)$ is linear in $(a-t)$ with slope $-\alpha$, with $\alpha$ interpreted as unit disutility of travel time; and it is also piecewise linear in $a$ in two segments with slopes $\beta$ and $-\gamma$, with $\beta$ and $\gamma$ interpreted as unit disutilities of early or late arrival. See Vickrey (1969) and Arnott et al. (1993); this is sometimes called the "bottleneck model" or the " $\alpha, \beta, \gamma$ model". 8

In the next theorem, we establish properties of the general Vickrey model that parallel those of our model as given in Theorem 1. We also state some additional results, which generalize results already known for the "bottleneck model".

The next theorem considers tolls that, following convention, are subtracted from the utility $V$. In the literature following Vickrey, utility would be interpreted as being in monetary units with the understanding that the complete utility function also contains an additive term equal to the consumption of the numeraire good. ${ }^{9}$ This interpretation would, however, create a problem when we later discuss our endogenous scheduling model from the perspective of the general Vickrey model, since numeraire consumption interacts explicitly with scheduling through $W$ in the endogenous scheduling model. We will instead interpret a toll that is subtracted from utility as being, simply, in utility units and we will call this a "utility toll". At the same time we define an equivalent money toll as the corresponding equivalent variation (we make this definition formal in the next section).

Theorem 2 (Properties of the general Vickrey model).

(a) In the general Vickrey bottleneck model described by utility $V(t, a)$, Nash equilibrium occurs when departures and arrivals take place during the interval

\footnotetext{
${ }^{8}$ Vickrey (1973) showed that his specification of scheduling utility in terms of utility rates is consistent with the more conventional $\alpha, \beta, \gamma$ utility specification. However we have restricted the Vickrey model slightly by requiring strict concavity, so that the $\alpha, \beta, \gamma$ utility specification is a limiting case rather than a special case of our "general" Vickrey model.Vickrey did not derive equilibria for his more general model, but only for the $\alpha, \beta, \gamma$ version.

${ }^{9}$ Vickrey (1973) calls his special case of $V(t, a)$ "value", thus enabling him to subtract money costs and tolls in determining welfare.
} 
$\left[t_{0}, t_{1}\right]$ determined uniquely by (5) and by

$$
\widetilde{V}\left(t_{0}\right)=\widetilde{V}\left(t_{1}\right)
$$

where $\widetilde{V}(t) \equiv V(t, t)$ is "undelayed utility," i.e. utility implied by a given schedule if there were no delays. We write this equilibrium utility level, which is a function of $\psi$, as

$$
\widetilde{\Delta}=\widetilde{\Delta}(\psi)=\widetilde{V}\left[t_{0}(\psi)\right] .
$$

(b) The marginal utility gain from a capacity improvement is

$$
\frac{\partial \widetilde{\Delta}}{\partial \psi}=-\frac{N}{\psi^{2}} \frac{\widetilde{V}^{\prime}\left(t_{0}\right) \widetilde{V}^{\prime}\left(t_{1}\right)}{\widetilde{V}^{\prime}\left(t_{0}\right)-\widetilde{V}^{\prime}\left(t_{1}\right)}>0 .
$$

(c) An optimal utility toll schedule $\widetilde{\tau}(t)$ satisfies

$$
\widetilde{V}(t)-\widetilde{\tau}(t)=\widetilde{V}\left(t_{0}\right)-\widetilde{\tau}\left(t_{0}\right) \quad \forall t \in\left[t_{0}, t_{1}\right]
$$

One such toll schedule also has $\widetilde{\tau}\left(t_{0}\right)=0$, in which case everyone receives the same utility (before any revenue distribution) as without the toll.

(d) The welfare gain from the toll schedule with $\widetilde{\tau}\left(t_{0}\right)=0$ described in part (c) is equal to the toll revenue (again in utility units), namely

$$
\psi \int_{t_{0}}^{t_{1}}(\widetilde{V}(t)-\widetilde{\Delta}) d t
$$

The intuition behind parts (a), (c), and (d) of the theorem is straightforward and well known within the special case usually called the "bottleneck model" (Arnott et al., 1993). Equilibrium implies that departures take place during an interval that is just long enough to allow all workers to pass the bottleneck; otherwise, someone could reduce scheduling cost without increasing queuing cost. The first and last commuters do not queue, since otherwise they could change departure time and thereby reduce their travel costs. This condition determines the start and end times of the departure interval, as a function of the utility $\widetilde{V}$ of those two commuters. For those in the interior of the departure interval, whose scheduling costs are smaller, utility is equalized by the existence of queuing costs, a condi- 
tion that in turn determines the departure rates needed to generate those queueing costs. From each individual's point of view, the motivation for departing at a time that yields positive scheduling costs is to reduce queuing, and the motivation for accepting some queuing is to reduce scheduling cost compared to that of the first and last commuters. The optimal toll alters departure times to prevent queuing from emerging, so that everyone's scheduling utility is the "undelayed"

utility $\widetilde{V}(t)$. It accomplishes this by equalizing the undelayed utility minus toll across departure times. The shape of the optimal toll is thus the same as the shape of undelayed utility without toll. The optimal toll includes an arbitrary constant $\widetilde{\tau}\left(t_{0}\right)$; when it is set to zero, the welfare gain from tolling consists of savings in queuing costs, which are exactly equal to the toll revenue.

\section{Vickrey meets endogenous scheduling preferences}

Individuals in our model have preferences defined only over leisure and consumption; they care indirectly about the timing of work trips only because it affects production at work and at home. Production is in turn affected by the scheduling of work trips of all other individuals through the agglomeration effects specified in our model.

However, a single individual, taking equilibrium as given, will appear to have preferences concerning the scheduling of his commute. This section will show that scheduling preferences like those specified in the general Vickrey model can appear to explain individual behavior when taking the equilibrium departure pattern as given, even if actual preferences are as we posit.

A naïve analyst observing an equilibrium departure pattern generated by our model may feel justified in applying the general Vickrey model to determine the effect of policies, since the general Vickrey model is able to generate exactly the observed equilibrium. However, a change in capacity or some other aggregate parameter will lead to a change in the equilibrium departure pattern and hence in the apparent scheduling preferences. Therefore such a naïve analyst will not be able to predict correctly the effects of a capacity change. A general result does not seem available concerning the direction of the mistakes such an analyst would 
make. ${ }^{10}$ Concerning the optimal toll, however, we can make a more definite statement: Theorem 4 shows that the naïve analyst would underestimate the welfare gain available from this toll.

Consider an individual in our endogenous scheduling model who, in Nash equilibrium, departs from home at time $t \geq t_{0}$ and arrives at work at time $a \leq t_{1}$. Inserting the appropriate portions of (3) and (4) into utility $U(H, W)$ shows that utility achieved is

$$
V(t, a)=U\left[\begin{array}{c}
t_{0}+\int_{0}^{t-t_{0}} g_{H}\left(1-\frac{R\left(s \mid t_{0}\right)}{N}\right) d s \\
\Omega-t_{0}-\frac{N}{\psi} \cdot\left[1-G_{W}(1)+G_{W}\left(\frac{\psi\left(a-t_{0}\right)}{N}\right)\right]
\end{array}\right] .
$$

We now consider the behavior of such an individual who regards the cumulative departure pattern $R\left(\cdot \mid t_{0}\right)$ as exogenous.

Theorem 3 Consider a single individual who is part of a Nash Equilibrium in our endogenous scheduling model. If this individual takes the cumulative departure pattern as exogenous, he or she would act according to a utility function (12), valid for all $t$ and $a$ in the open interval $\left(t_{0}, t_{1}\right)$. This function meets the requirements of the general Vickrey model.

We now consider optimal tolling in the endogenous scheduling model. In this model, numeraire consumption arises from production $W$, rather than being added onto the scheduling utility ad hoc. Given values of $H$ and $W$, we can translate a "utility toll" $\tau$ payment into a corresponding to money toll payment $T$ through

$$
\tau=U(H, W)-U(H, W-T)
$$

and a solution exists uniquely since the last term is monotonically strictly decreasing in $T$. Given profiles $H(t)$ and $W(t)$, once we have computed the utility toll schedule $\tau(t)$ we can solve (13) to find $T(t)$.

We must also consider what we mean by optimality. Any redistribution scheme for toll revenues would alter the balance of marginal utilities of work and leisure

\footnotetext{
${ }^{10}$ The numerical examples in the next section show situations where the general Vickrey model would lead to underestimates of the benefit of capacity expansion compared to the true model. We have not been able to find conditions under which such underestimation would always occur.
} 
and hence affect the equilibrium. In general, then, it is difficult or perhaps impossible to determine a toll that maximizes utility including a redistribution of revenues. We avoid the issue by considering a situation where toll revenues accrue to an outside party which has some social value, so social welfare can be written as

$$
\omega=\omega(U, Y)
$$

where $U$ is average utility and $Y$ is aggregate toll revenues. We assume both derivatives of $\omega$ are positive, and that the derivative with respect to toll revenue is not so large as to make it optimal to extract revenue by setting tolls at a level that would leave capacity less than fully utilized during the commute.

We are now able to provide a strong result about the effect of the optimal toll in the true model compared to what a naïve analyst would predict.

Theorem 4 (Optimal toll) Suppose the untolled equilibrium results in travel during interval $\left[t_{0}, t_{1}\right]$, so that the Vickrey model predicts an optimal toll that is zero at $t_{0}$ and $t_{1}$ and positive elsewhere on $\left[t_{0}, t_{1}\right]$. Under endogenous scheduling preferences:

(a) The following utility toll produces a larger welfare gain than the maximum possible gain predicted by the general Vickrey model:

$$
\hat{\tau}(t)=\hat{V}(t, t)-\widetilde{V}\left(t_{0}, t_{0}\right)
$$

where $\widetilde{V}(\cdot)$ is the Vickrey utility calculated from the observed untolled equilibrium, and $\hat{V}(\cdot)$ is the true utility when travelers depart at rate $\psi$ starting at $t_{0}$. This toll is larger than the Vickrey toll on $\left(t_{0}, t_{1}\right]$.

(b) The optimal toll produces a welfare gain that is at least as large as that from the toll given in (14).

Part (b) of the theorem follows directly from part (a). The proof of the theorem (Appendix A) considers the toll (14), which in our setup is not optimal but is an improvement over the Vickrey toll. It removes queueing (in the true model) while maintaining the first equilibrium departure time to be the same as in the equilibrium solution to the Vickrey model. This toll is larger than in the Vickrey model because it takes into account that workers depart later than in Nash equilib- 
rium and therefore that agglomeration leads to a higher production of leisure. The agglomeration effect is not present in the Vickrey model and hence the Vickrey model predicts a smaller efficiency gain. This toll is not optimal because it does not re-optimize the initial departure time $t_{0}$. The finding that the toll that removes congestion in the true model is larger than in the Vickrey model model implies also that the Vickrey toll (10) would fail to remove all congestion (in contrast to its intent).

We have not found an analytical solution for the fully optimal toll. We conjecture that if one exists, it contains terms related to the Pigou subsidies correcting the positive externalities of agglomeration, integrated over the period of travel. In the numerical example in the next section, we determine the optimal toll numerically and find that in contrast to the Vickrey toll, it can shift the first departure time $t_{0}$ substantially from its value in the untolled equilibrium. Thus, it appears that agglomeration effects may create a potential for substantial efficiency improvements beyond those from eliminating queueing.

We now consider the marginal benefits (in terms of utility) from adding capacity. It will be convenient to introduce the following notation for utilities and marginal utilities (with respect to the arguments of the utility functions) for the first and last travelers, given that the first departs at time $t_{0}$.

\section{Notation 1}

$$
\begin{aligned}
U^{0} & =U\left[t_{0}, \Omega-t_{0}-\frac{N}{\psi}+\frac{N}{\psi} G_{W}(1)\right] \\
U^{1} & =U\left[t_{0}+\int_{0}^{\frac{N}{\psi}} g_{H}\left(1-\frac{R\left(s \mid t_{0}\right)}{N}\right) d s, \Omega-t_{0}-\frac{N}{\psi}\right] \\
U_{H}^{0} & =U_{H}\left[t_{0}, \Omega-t_{0}-\frac{N}{\psi}+\frac{N}{\psi} G_{W}(1)\right]
\end{aligned}
$$

The derivatives $U_{H}^{1}, U_{W}^{0}$, and $U_{W}^{1}$ are defined similarly.

Lemma 2 Suppose the true model is our model of endogenous scheduling preferences. Then

(a) The generalized Vickrey utilities for the first and last traveler vary with $t_{0}$ according to $\widetilde{V}^{\prime}\left(t_{0}\right)=U_{H}^{0}$ and $\widetilde{V}^{\prime}\left(t_{1}\right)=-U_{W}^{1}$; 
(b) Interpreting the equilibrium as coming from the general Vickrey model, utility would be thought to vary with capacity according to:

$$
\frac{\partial \widetilde{\Delta}}{\partial \psi}=\frac{N}{\psi^{2}} \cdot \frac{U_{H}^{0} U_{W}^{1}}{U_{H}^{0}+U_{W}^{1}}
$$

(c) The true variation of utility with capacity expansion is:

$$
\frac{\partial \Delta}{\partial \psi}=\left(U_{H}^{0}-U_{W}^{0}\right) \frac{\partial t_{0}}{\partial \psi}+U_{W}^{0} \frac{N}{\psi^{2}}\left[1-G_{W}(1)\right] .
$$

Equation (17) states that the marginal utility of capacity arises from two sources: (i) the utility change to the first traveler from production and leisure as $t_{0}$ is shifted, and (ii) the change in production for the first traveler due to more people being at work at each time after $t_{0}$. These marginal values of capacity expansion are calculated numerically in the next section.

\section{Numerical example}

In this section we illustrate the workings of the model by solving it for particular functional forms and parameter values. Mostly we use this tool to explore how the model behaves under quite wide variations in parameters, in order to depict the range of its possibilities for explaining various observable patterns. Thus the first five simulations portray fairly extreme situations. In our last simulation, by contrast, we calibrate so as to produce features typical of a realistic city. In each case, we compute the full social optimum (thus going beyond the results of Theorem 4) and we compute the extent of the errors that would occur from using the Vickrey model for policy prescriptions if behavior is affected by agglomeration as depicted by our model.

For our main set of runs, we set $\Omega=10$ (interpreted as a morning lasting from $A=2: 00$ a.m. to $A+\Omega=12: 00$ ). We set $N=2$ and $\psi=1$; thus the congested period is exactly two hours long. (We then vary $\psi$ in order to calculate the marginal benefit of capacity.) We also take the utility function to be Cobb- 
Douglas, and the agglomeration functions to be simple power functions:

$$
\begin{aligned}
U(H, W) & =\alpha \ln H+\ln W \\
g_{H}(x) & =x^{\pi_{H}} \\
g_{W}(x) & =x^{\pi_{W}}
\end{aligned}
$$

with $\pi_{W}, \pi_{H}>0$. We require $\pi_{H}<1$ because otherwise, as we show in Appendix $\mathrm{B}$, our simulation yields no solution.

Given some initial departure time $t_{0}$, equations (3) and (4) then give the following analytical expressions for effective leisure and work production for a traveler departing at any time $\mathrm{t} \in\left[t_{0}, t_{0}+N / \psi\right]$ :

$$
\begin{gathered}
H(t)=t_{0}+\int_{0}^{t_{d}}\left(1-\frac{R\left(t^{\prime} \mid t_{0}\right)}{N}\right)^{\pi_{H}} d t^{\prime} \\
W[a(t)]=\Omega-t_{0}-\frac{1}{\psi}\left(\frac{\pi_{W}+R\left(t_{d} \mid t_{0}\right)^{\pi_{W}+1}}{\pi_{W}+1}\right),
\end{gathered}
$$

where $t_{d}=t-t_{0}$, and where $a(t)=t_{0}+R\left(t_{d} \mid t_{0}\right) / \psi$ is the arrival time of thi traveler. If we substitute these expressions into the utility function in (18), we get utility as a function of departure time, which we write as $\widetilde{U}(t)$.

Our problem is twofold: (a) to find the cumulative departure function $R\left(t_{d} \mid t_{0}\right)$ that makes $\widetilde{U}(t)$ constant; and (b) to find the initial departure time $t_{0}$ that ensures everyone travels, i.e. $R\left[(N / \psi) \mid t_{0}\right]=1$. Task (a) is accomplished by setting the total derivative of $\widetilde{U}(t)$ equal to zero, yielding:

$$
\frac{\alpha}{H(t)}[1-R(\cdot)]^{\pi_{H}}-\frac{1}{W[a(t)]} R(\cdot)^{\pi_{W}} \frac{\rho(\cdot)}{\psi}=0
$$

where $(\cdot)$ is shorthand for $\left(t-t_{0} \mid t_{0}\right)$, and $\rho$ is the derivative of $R$. We can rewrite this condition as

$$
\rho(\cdot)=\frac{\psi \alpha W[a(t)]}{H(t)} \frac{[1-R(\cdot)]^{\pi_{H}}}{R(\cdot)^{\pi_{W}}} .
$$

Since $\rho=R^{\prime}$, this is a differential equation in $R$, which can be solved numerically given a value of $t_{0}$.

For task (b), an outer iterative procedure is employed to find the unique value 
Table 1: Simulation results

\begin{tabular}{|l|r|r|r|r|r|r|}
\hline Simulation number & 1 & 2 & 3 & 4 & 5 & 6 \\
\hline Parameters: & & & & & & \\
Time universe & & & & & & \\
$\quad$ (Hours past midnight) & $2-12$ & $2-12$ & $2-12$ & $2-12$ & $2-12$ & $0-12$ \\
alpha & 1 & 1 & 1 & 1 & 0.5 & 2 \\
$\pi_{H}$ & 0.5 & 0.5 & 0.5 & 0.75 & 0.5 & 0.1 \\
$\pi_{W}$ & 0.5 & 1 & 3 & 0.5 & 0.5 & 0.1 \\
$N / \psi$ (duration of peak hours) & 2 & 2 & 2 & 2 & 2 & 2 \\
\hline Nash equilibrium: & & & & & & \\
(1) $t_{0}$ (hours past midnight) & 4.95 & 5.28 & 6.08 & 3.64 & 3.64 & 6.85 \\
(2) $t_{d}$ at max travel time & 5.57 & 5.79 & 6.38 & 4.14 & 4.25 & 7.70 \\
(3) Max travel time (hr) & 0.83 & 0.96 & 1.25 & 1.16 & 0.85 & 0.31 \\
(4) Average travel time (hr) & 0.56 & 0.64 & 0.79 & 0.74 & 0.58 & 0.21 \\
(5) $R\left(t_{d}=\left(t_{1}-t_{0}\right) / 10\right)$ & 0.41 & 0.51 & 0.72 & 0.58 & 0.42 & 0.18 \\
(6) Vickrey/Actual $d U / d \psi$ & 0.94 & 0.83 & 0.69 & 0.73 & 1.00 & 2.15 \\
\hline Social optimum: & & & & & & \\
(7) $t_{0}$ (hours past midnight) & 6.00 & 5.94 & 5.82 & 6.03 & 4.43 & 6.95 \\
(8) $\left(\Delta U_{\text {min }}\right.$ ) & 0.13 & 0.06 & -0.01 & 0.49 & 0.09 & 0.01 \\
(9) Average toll & 0.16 & 0.24 & 0.34 & 0.22 & 0.17 & 0.10 \\
\hline
\end{tabular}

of $t_{0}$ for which total cumulative departures are exactly $N$. More details are in Appendix B. The value is unique because $H(t)$ and $W(t)$ are increasing and decreasing, respectively, in $t_{0}$, so that $\rho$ in (21) will be smaller the larger is $t_{0}$; this is discussed in the proof of unique existence of Nash equilibrium in Appendix A.

Results are shown in rows labeled (1)-(5) in Table 1. Consider simulation $\# 1$, for which $\alpha=1$ and $\pi_{H}=\pi_{W}=1 / 2$. The congested period extends between times 4.95 and 6.95 in our 10-hour "morning"; thus it is shifted earlier by 1.05 hours compared to one that is centered during the morning (which would be from 6:00 to 8:00). Forty-one percent of travelers depart during the first twelve minutes (row 5), resulting in congestion reaching a peak travel time of 0.83 hours. Cumulative departures and arrivals are shown as the two left-most curves in the top panel of Figure 3.

By varying capacity $\psi$, we can calculate numerically the derivative in equation (17) and thus compute the marginal value of capacity in untolled equilibrium. We compare this with the marginal value predicted by the Vickrey analysis, namely 


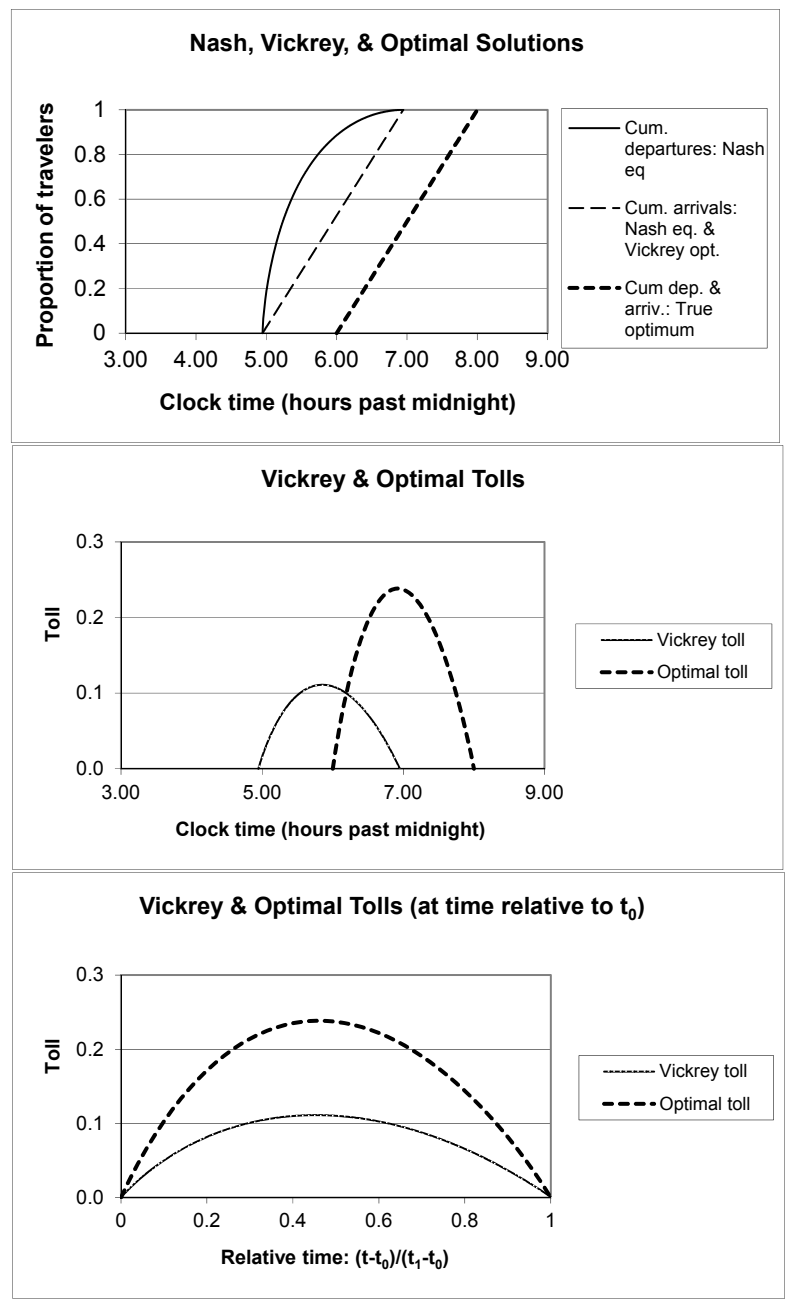

Figure 3: Graphs for Simulation \#1 
equation (16). Row (6) of Table 1 shows the ratio of these two calculations: we see that the Vickrey model underpredicts benefits in most cases, but overpredicts them when agglomeration is small (Simulation \#6). ${ }^{11}$

We also compute the Vickrey toll $\widetilde{\tau}(t)$ using (10). Its two terms can be calculated, using (12) and (18), as

$$
\begin{aligned}
\widetilde{V}(t) & =\alpha \ln [H(t)]+\ln [W(a(t))] \\
-\widetilde{V}\left(t_{0}\right) & =-\alpha \ln \left(t_{0}\right)-\ln \left(\Omega-t_{0}-\frac{N}{\psi} \cdot \frac{\pi_{W}}{\pi_{W}+1}\right) .
\end{aligned}
$$

Recall that the Nash equilibrium departure pattern $R(\cdot)$ is chosen to make $\widetilde{V}\left(t_{1}\right)=$ $\widetilde{V}\left(t_{0}\right)$; thus the Vickrey toll begins and ends at a value of zero, because the Vickrey analyst assumes that the first and last travelers (who avoid queueing) care only about their schedules and therefore achieve the same utility as without the toll. The resulting toll for simulation \#1 is shown as the solid curve in the second panel of Figure 3 ; it rises rapidly after time $t_{0}$ and then falls more gradually from its peak value.

Finally, in each case we have carried out a simulation of the social optimum. It is achieved by applying the optimal toll which, like the Vickrey toll, is set to maintain a constant departure rate $\rho=\psi$. In contrast to the Vickrey toll, however, the optimal toll actually achieves this departure rate. In this calculation, $t_{0}$ is set so as to maximize the average of travelers' gross utilities (i.e., their utilities before paying tolls). That value is

$$
\widehat{\bar{U}}(t)=\max _{t_{0}} \psi \int_{t_{0}}^{t_{0}+1 / \psi} U[\widehat{H}(t), \widehat{W}(t)] d t
$$

where $\widehat{H}(t)$ is computed numerically from (3) but with $R\left(t_{d} \mid t_{0}\right)=\psi t_{d}$, and where $\widehat{W}(t)$ is computed as before except now $t_{0}$ takes a different value. Details of calculating (22) are given in Appendix B. Since $U[\widehat{H}(t), \widehat{W}(t)]$ is a concave

\footnotetext{
${ }^{11}$ The more detailed calculations show that both terms in (17) are of similar size except in Simulation \#3, in which the second dominates - presumably due to the high importance of work agglomeration in this simulation.
} 
function of $t$, it attains its minimal value $\widehat{U}_{\min }$ at either $t_{0}$ or $t_{1}$; we fix the toll $\hat{\tau}$ to be zero at this point, which implies that the $\hat{\tau}$ is non-negative at all times in $\left[t_{0}, t_{1}\right]$. With an optimum toll, all travelers achieve the same utility, and this is equal to the net utility (utility minus toll) of any traveler and in particular it is equal to $\widehat{U}_{\min }$. We report on $\widehat{U}_{\min }$ in our simulation results.

Rows (7)-(9) provide more results concerning the social optimum. The second panel of Figure 3 shows the optimal money toll (for Simulation \#1), which for this example is given by the following analytical solution to (13):

$$
T(t)=W(a(t)) \cdot\left(1-e^{-\tau(t)}\right)
$$

In Simulation \#1, the optimal first departure time $t_{0}$ is exactly 6.0 (6:00 a.m.), so that the two-hour departure interval is centered in the available time universe [2:00, 12:00]. This result reflects the symmetry of leisure and work in this simulation (since $\pi_{H}=\pi_{W}$ and $\alpha=1$ ). This optimal value of $t_{0}$ is substantially later than the Nash equilibrium value of 4.95 . The symmetry of this simulation causes the optimal toll to be perfectly symmetric about the midpoint of the morning (7.00). The Vickrey starts later and reaches larger values than the optimal toll, as seen in the second and third panels of the figure.

Simulations \#2 and \#3 explore successively larger values of $\pi_{W}$, whereas simulation \#4 looks at a larger value of $\pi_{H}$. Simulations \#5 and \#6 consider a smaller and larger value, respectively, for $\alpha$.

The shape of the equilibrium departure pattern, as a function of $t-t_{0}$, is governed mainly by the relative values of $\pi_{H}$ and $\pi_{W}$. Smaller values result in the least congestion and the lowest fraction of travelers who depart early in the departure interval. The location of the rush hour within the available time shifts in opposite directions depending on which agglomeration is increased: earlier for increasing $\pi_{H}$ and later for increasing $\pi_{W}$. Increasing $\alpha$, the weight on household production in the utility function, makes little difference to the pattern of departures or to the amount of travel delay; but it shifts the start of the departure interval to a later time in order to restore balance between $U_{H}$ and $U_{W}$.

Increasing $\pi_{W}$, which controls agglomeration at work, causes two changes (simulations \#2 and \#3). First, the optimal first departure time shifts earlier, 
whereas the Nash equilibrium value shifts later. In simulation \#3, these opposing shifts are so strong that the socially optimal rush hour starts earlier than the Nash equilibrium rush hour, in contrast to all other simulations. Second, the utility difference for the last traveler, relative to the first, becomes positive, meaning the optimal toll is now higher for a departure at time $t_{1}$ than one at time $t_{0}$ (not shown in the table). These effects are reversed when it is $\pi_{H}$ rather than $\pi_{W}$ that is raised (simulation \#4). Note that in this latter simulation, the higher home agglomeration parameter causes the optimal commuting interval (always two hours in length) to occur slightly later than in the case with symmetric parameters (simulation \#1), due to a greater social value of letting everyone spend time at home together; whereas the Nash equilibrium commuting interval occurs considerably earlier due to more congestion.

Simulations \#5 changes the relative importance of work through parameter $\alpha$ in the utility function. The first departure time is quite sensitive to $\alpha$, but remains consistently about an hour earlier in equilibrium than in the optimum. Decreasing $\alpha$ shifts first departure times earlier, allowing people to spend more time at work.

The final simulation (\#6) is designed to produce a more realistic pattern of congestion, lasting from 6:51 a.m. to 8:51 a.m. and reaching maximum congestion delay of 19 minutes. We achieve this result by extending the considered time period to $\Omega=12$ (interpreted as from midnight to noon), choosing a high value of $\alpha$ so that people choose to spend longer at home, and choosing small values for agglomeration parameters so that people don't tolerate so much congestion. In this simulation, most differences between the Vickrey and actual model are not nearly as pronounced: first departure time differs by just six minutes, and the utility gain from tolling differs by about ten percent. However, in this simulation the Vickrey model drastically errs in its cost-benefit analysis of a marginal capacity increase in the absence of a toll, overstating the true value by 115 percent as seen in row (6).

For each simulation we calculate $\widehat{U}_{\text {min }}$, the minimum gross (i.e. pre-toll) utility obtained in social optimum. As already noted, it is the utility of the traveler who is charged a toll of zero, which is either the first or the last traveler. The difference between $\widehat{U}_{\min }$ and the utility obtained by travelers in Nash equilibrium is therefore the net (post-toll) utility gain realized by all travelers. Row (8) shows that this 
difference is positive in most cases, indicating that the optimal toll leads to a strict Pareto improvement even if toll revenues are not returned to travelers. However, simulation \#3 shows a contrary case, where travelers will lose (very slightly) in the social optimum, compared to Nash equilibrium, if toll revenues are not returned. Both of these results are in contrast with the Vickrey model, which predicts zero change in gross utility.

Figures 3-5 present graphs of the departure patterns $R(\cdot)$ and of the Vickrey and optimal tolls, for selected simulations. In all three cases, the Vickrey toll differs greatly from the optimal toll, though for different reasons. In simulation \#3, with high work agglomeration, the Vickrey analyst predicts the optimal departure pattern quite closely, but is wildly off on the size and shape of the toll that will achieve it. In simulation \#4, with high leisure agglomeration, the Vickrey analyst gets the shape of the toll roughly correct, but its magnitude is too high and its position in time is much too early, resulting in people spending too little time at home and thus not taking advantage of the high marginal utility of leisure time with these parameters.

\section{Conclusion}

This paper has presented a dynamic model of traffic congestion in which scheduling preferences arise endogenously. A naïve Vickrey-like analyst - observing one equilibrium and assuming scheduling preferences to be exogenously given would find that to be in accordance with his model. But because he ignores the positive temporal agglomeration externalities associated with being at home or at work, this analyst will underestimate the benefits of a toll that reduces queueing during the commute and will make potentially very large errors in predicting the effect of policies such as capacity expansion and tolling. For some parameter sets, such an analyst would apply a toll schedule and/or aim for a departure pattern that is quite far removed from the optimal one. So a policy conclusion is that a gradual approach to introducing a policy such as road pricing is advisable, since that allows the consequences to be observed as one goes along.

The general conclusions depend on the result of ignoring an externality (agglomeration) and therefore seem likely to be quite robust. Hence we may expect 


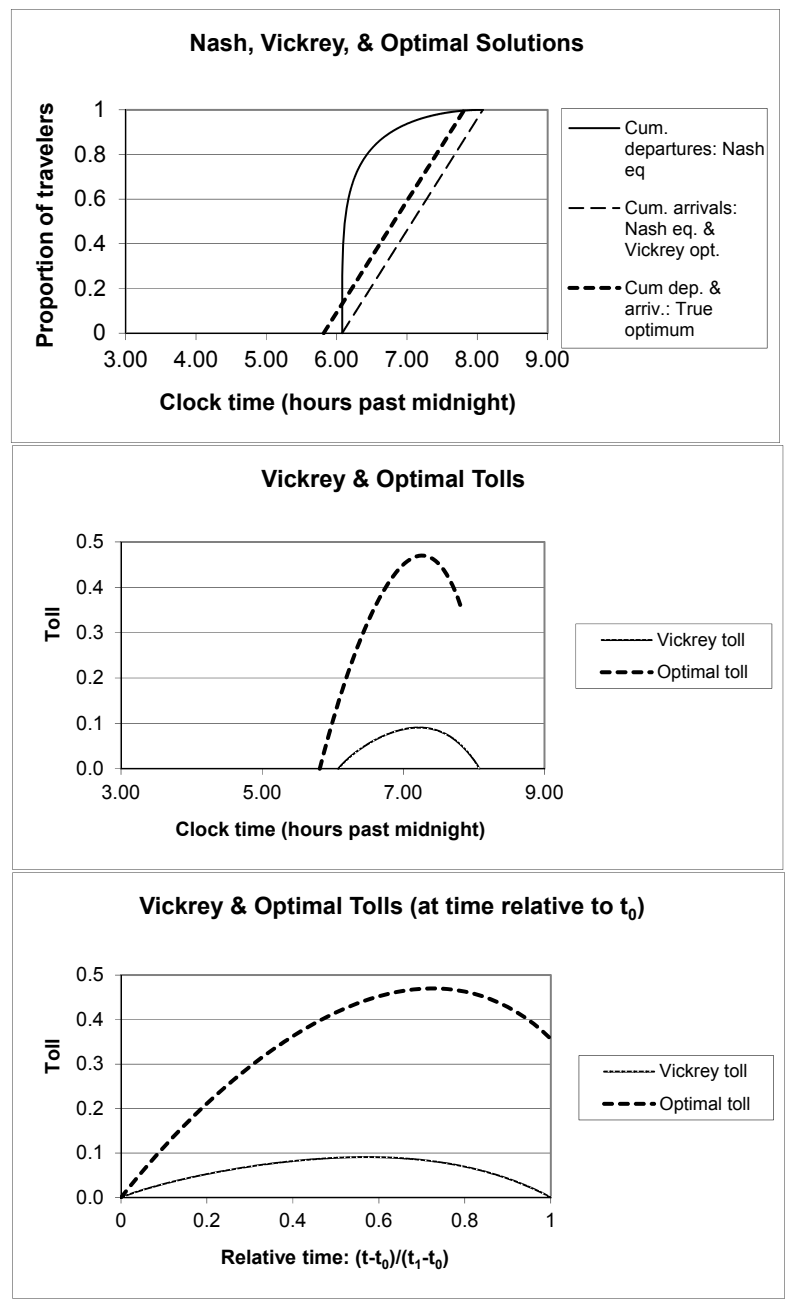

Figure 4: Graphs for Simulation \#3 


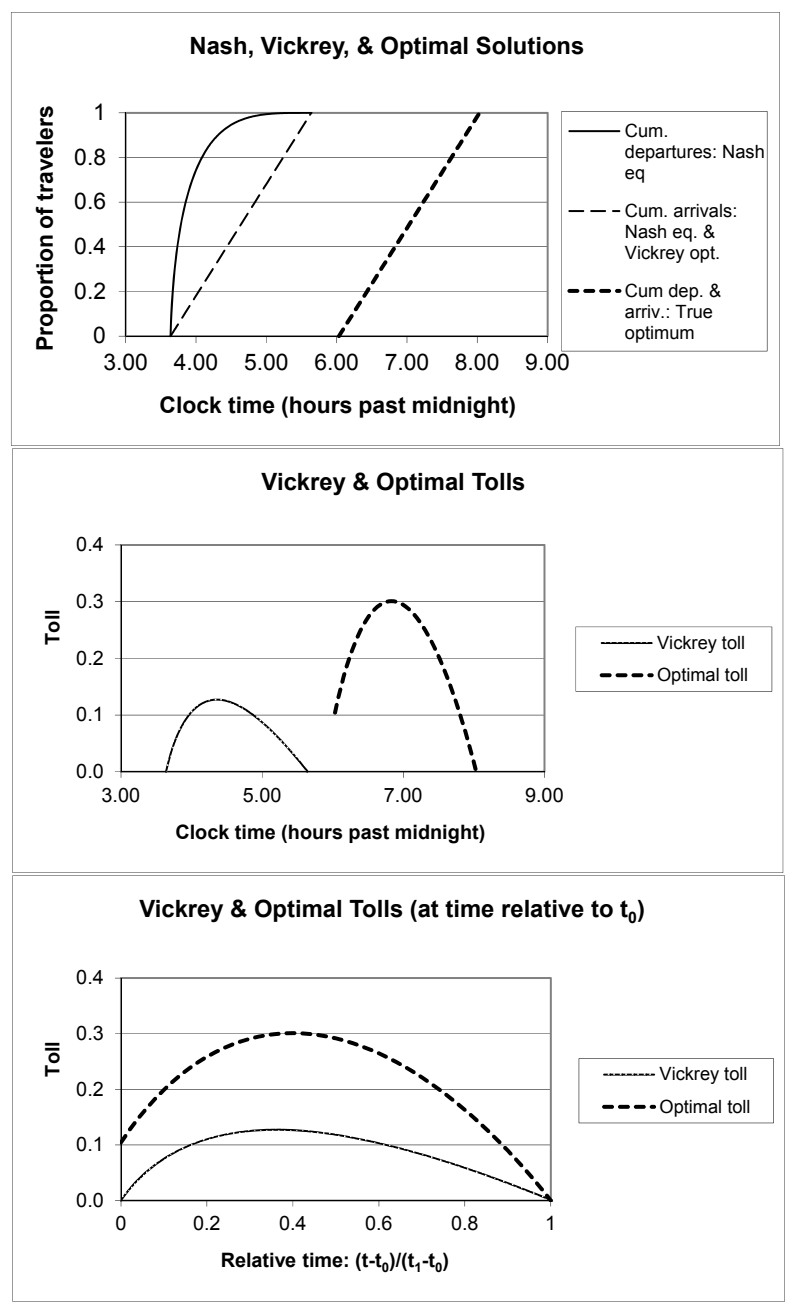

Figure 5: Graphs for Simulation \#4 
that these conclusions would survive if we relax the assumptions that we have made for the sake of analytical tractability.

We have assumed, first, that the productivity of effective leisure and work depend solely on the share of workers at home and at work at each point in time. We have furthermore assumed that a worker working alone is completely unproductive and similarly that a worker derives no effective leisure from solitary leisure time. The essential mechanism here is the positive externality associated with being at home or at work. As long as this remains, it seems possible to relax assumptions regarding productivity without affecting the validity of our general conclusions. Indeed, we found through simulations not reported here that even with no agglomeration (i.e. effective leisure and production are not dependent on other people), concave utility produces a unique single-peaked equilibrium. ${ }^{12}$

Second, we have made assumptions that allow us to ignore the evening commute. The model describes people as staying at work until some common time and is silent about what happens thereafter. Incorporating the evening commute would not affect the conclusions that depend on a Vickrey-like analyst ignoring agglomeration externalities.

Third, we have assumed that the demand for travel is completely inelastic. As has been noted earlier, this reduces the importance of the assumption that productivity depends on the share of all workers present at home or at work. Allowing for elastic demand would likely enable us to separate the problem into two parts, similarly to Arnott et al. (1993), including removing the indeterminacy on the level of the toll. However we would then have to deal explicitly with city-size agglomeration effects.

Fourth, the paper assumes that individuals are identical. Allowing for heterogeneity would raise the issue of how workers sort in equilibrium, ie. in which sequence they pass the bottleneck. The presence of heterogeneity would add a possibility for efficiency gain from a toll that causes workers to sort in a more efficient way. It seems likely that our general conclusions would remain valid due to the remaining central premise that positive agglomeration externalities shape

\footnotetext{
${ }^{12} \mathrm{We}$ also verified through simulations that if we eliminate agglomeration and make the utility function close to being flat, congestion is essentially eliminated as people choose travel schedules so that their departure rate exceeds capacity by only a small amount.
} 
scheduling preferences.

There are no firms in our model. Firms could seek to internalize agglomeration externalities by paying a wage that depends on the time a worker arrives at the work place (instead of the integral of the productivity rate over time spent at work, as implicitly assumed here). It would be an interesting extension of the present analysis to include such aspects of firm behavior, but probably also very difficult except in the trivial case of a single firm fully internalizing the externality.

The model with endogenous scheduling preferences generates an equilibrium that is indistinguishable from a model with exogenous scheduling preferences. It is hence not possible to falsify the latter model using only observation of individual choices in a single equilibrium; rather, in order to identify endogeneity, it is necessary to compare different equilibria. It may be possible to employ such an identification strategy empirically, for example by using capacity expansion or the introduction of a road pricing scheme as an exogenous instrument in an empirical investigation explaining variations in the temporal shape of the morning peak.

Furthermore, by relaxing certain of these assumptions, it could become possible to test the importance of endogenous scheduling preferences empirically. For example, suppose we relax the first assumption and allow for exogenous factors affecting work productivity or utility from leisure time. These would form an alternative explanation of peaking, and by observing variations in those factors one could see how much differences in peaking can be explained in this way. For example, the duration of daylight varies both by latitude within a given season, and by season within a given location that is not close to the equator. If daylight has an important effect, one should observe resulting variations in the extent of peaking in commuting due to this external force acting to make scheduling preferences exogenous. $^{13}$

\section{References}

Anas, A. and Kim, I. (1996) General Equilibrium Models of Polycentric Urban Land Use with Endogenous Congestion and Job Agglomeration Journal of Ur-

\footnotetext{
${ }^{13} \mathrm{We}$ are grateful to an anonymous referee for suggesting this example.
} 
ban Economics 40(2), 232-256.

Arnott, R. A. (2007) Congestion tolling with agglomeration externalities Journal of Urban Economics 62(2), 187-203.

Arnott, R., de Palma, A. and Lindsey, R. (1990) Economics of a bottleneck Journal of Urban Economics 27(1), 111-130.

Arnott, R., de Palma, A. and Lindsey, R. (1993) A structural model of peak-period congestion: A traffic bottleneck with elastic demand American Economic Review 83(1), 161-179.

Arnott, R., de Palma, A. and Lindsey, R. (1998) Recent developments in the bottleneck model in K. Button and E. T. Verhoef (eds), Road Pricing, Traffic Congestion and the Environment: Issues of Efficiency and Social Feasibility Edward Elgar Cheltenham, UK pp. 79-110.

Chinitz, B. (1961) Contrasts in Agglomeration: New York and Pittsburgh The American Economic Review 51(2), 279-289.

Cohen, Y. (1987) Commuter welfare under peak-period congestion tolls: Who gains and who loses? International Journal Transport Economics 14, 238-266.

de Palma, A. and Fosgerau, M. (2011) Dynamic and static congestion models: a review in A. de Palma, R. Lindsey, E. Quinet and R. Vickerman (eds), A Handbook of Transport Economics Edward Elgar chapter 9.

Duranton, G. and Puga, D. (2004) Micro-foundations of urban agglomeration economies in J. Vernon Henderson and Jacques-Fran Ã ğois Thisse (ed.), Handbook of Regional and Urban Economics Vol. Volume 4 of Cities and Geography Elsevier pp. 2063-2117.

Fosgerau, M. and Engelson, L. (2011) The value of travel time variance Transportation Research Part B: Methodological 45(1), 1-8.

Fujita, M. and Ogawa, H. (1982) Multiple equilibria and structural transition of non-monocentric urban configurations Regional Science and Urban Economics 12(2), 161-196. 
Glaeser, E. L. and Gottlieb, J. D. (2009) The Wealth of Cities: Agglomeration Economies and Spatial Equilibrium in the United States Journal of Economic Literature 47(4), 983-1028.

Glaeser, E. L., Kolko, J. and Saiz, A. (2001) Consumer city Journal of Economic Geography 1(1), 27-50.

Gutierrez-i Puigarnau, E. and Van Ommeren, J. N. (2012) Start Time and Worker Compensation Implications for Staggered-Hours Programmes Journal of Transport Economics and Policy 46(2), 205-220.

Helsley, R. W. and Sullivan, A. M. (1991) Urban subcenter formation Regional Science and Urban Economics 21(2), 255-275.

Henderson, J. V. (1981) The economics of staggered work hours Journal of Urban Economics 9, 349-364.

Hendrickson, C. and Kocur, G. (1981) Schedule Delay and Departure Time Decisions in a Deterministic Model Transportation Science 15(1), 62-77.

Krugman, P. (1991) Increasing Returns and Economic Geography Journal of Political Economy 99(3), 483-499.

Lindsey, R. (2004) Existence, Uniqueness, and Trip Cost Function Properties of User Equilibrium in the Bottleneck Model with Multiple User Classes Transportation Science 38(3), 293-314.

Lucas, R. E. and Rossi-Hansberg, E. (2002) On the Internal Structure of Cities Econometrica 70(4), 1445-1476.

Manski, C. F. (2000) Economic Analysis of Social Interactions The Journal of Economic Perspectives 14(3), 115-136.

Newell, G. (1987) The Morning Commute for Nonidentical Travelers Transportation Science 21(2), 74-88.

Rosenthal, S. S. and Strange, W. C. (2004) Evidence on the nature and sources of agglomeration economies in J. V. Henderson and J. Thisse (eds), Cities and Geography Vol. 4 Elsevier pp. 2119-2171. 
Scott, A. J. (1988) Metropolis: from the division of labor to urban form University of California Press.

Small, K. A. and Chu, X. (2003) Hypercongestion Journal of Transport Economics and Policy 37, 319-352.

Small, K. A. and Verhoef, E. T. (2007) Urban transportation economics Routledge London and New York.

Starrett, D. A. (1974) Principles of optimal location in a large homogeneous area Journal of Economic Theory 9(4), 418-448.

Tabuchi, T. and Yoshida, A. (2000) Separating Urban Agglomeration Economies in Consumption and Production Journal of Urban Economics 48(1), 70-84.

Tseng, Y. Y. and Verhoef, E. T. (2008) Value of time by time of day: A stated-preference study Transportation Research Part B: Methodological 42(78), 607-618.

van den Berg, V. and Verhoef, E. T. (2011) Winning or losing from dynamic bottleneck congestion pricing?: The distributional effects of road pricing with heterogeneity in values of time and schedule delay Journal of Public Economics 95(7-8), 983-992.

Vickrey, W. (1969) Congestion theory and transport investment American Economic Review 59(2), 251-260.

Vickrey, W. (1973) Pricing, metering, and efficiently using urban transportation facilities Highway Research Record 476, 36-48.

Vovsha, P. and Bradley, M. (2004) Hybrid Discrete Choice Departure-Time and Duration Model for Scheduling Travel tours Transportation Research Record 1894, 44-56.

Wilson, P. W. (1988) Wage variation resulting from staggered work hours Journal of Urban Economics 24(1), 9-26. 


\section{A Proofs}

Proof of Lemma 1. Since the queue exists throughout $I$, and queueing time constitutes all travel time, the queue discharge rate $\psi$ is also the rate of arrivals at work. Then for any $t_{d} \in[0, N / \psi]$ we have $N_{H}\left(t_{0}+t_{d}\right)=N-R\left(t_{d} \mid t_{0}\right)$ and $N_{W}\left(t_{0}+t_{d}\right)=\psi t_{d}$. The expression for $H$ then follows immediately from (2); note that it is constant for all $t \geq t_{1}$. The expression for $W$ follows by calculating (1), evaluated for each of the three possible cases shown in (4).

Next, consider derivatives. First, $H^{\prime}(t)=1$ for $t \leq t_{0}$. For $t \geq t_{0}, H^{\prime}(t)=$ $g_{H}\left(1-\frac{R\left(t-t_{0} \mid t_{0}\right)}{N}\right)$, which is strictly positive until time $t_{1}$ and zero afterwards the latter because $R\left(s-t_{0} \mid t_{0}\right)=N$ for $s \geq t_{1}$. It is also continuous at $t_{1}$ because $g_{H}\left(1-\frac{R\left(t_{1}-t_{0} \mid t_{0}\right)}{N}\right)=g_{H}(0)=0$ by the definition of $t_{1}$ and the properties of $g_{H}(\cdot)$. Next, $H^{\prime \prime}(t)=-g_{H}^{\prime}\left(1-\frac{R\left(t-t_{0} \mid t_{0}\right)}{N}\right) \cdot \frac{\rho\left(t-t_{0} \mid t_{0}\right)}{N}$, from which we see $H^{\prime \prime}$ $<0$ for $t \in \operatorname{int}(I)$ and $H^{\prime \prime}=0$ outside $I$ - the latter because $N_{H}$ is constant there and thus so is $g_{H}$.

Similarly, $W^{\prime}(a)=0$ for $a<t_{0}, W^{\prime}(a)=-g_{W}\left[\psi \cdot\left(a-t_{0}\right) / N\right]$ for $a \in$ $I$, and $W^{\prime}(a)=-1$ for $a>t_{1}$; note $W^{\prime}$ is continuous everywhere because $\psi \cdot\left(t_{1}-t_{0}\right) / N=1$ (by the definition of $\left.t_{1}\right)$ and $g_{W}(1)=1$. Furthermore, $W^{\prime \prime}(a)=-(\psi / N) g_{W}^{\prime}\left[\psi \cdot\left(a-t_{0}\right) / N\right]<0$ for $a \in \operatorname{int}(I)$ and $W^{\prime \prime}=0$ outside $I$. Because $H, W, H^{\prime}$, and $W^{\prime}$ are all continuous at the boundaries of $I$, and $H^{\prime}$ and $W^{\prime}$ are non-increasing there, concavity holds at these boundaries as well.

Proof of Theorem 1. We need to show that Nash equilibrium exists uniquely, and that in Nash equilibrium the departure rate is strictly decreasing in time. Our strategy is to write utility as a function of departure time, measured as time after a given initial departure time, and to consider the implications of meeting a "total population" condition that the departure rate must integrate to $N$, the total number of workers over a duration of $N / \psi$ time units. We do this by defining a function $\bar{d}(t)$ relating the last departure time to the first departure time so as to meet the total population condition. We show that as $t$ covers its allowed values, $\bar{d}(t)$ first falls short of, then exceeds, the value $N / \psi$ that represents the minimum time over which the bottleneck can deliver travelers to their destination. By continuity there must then be at least one value of $t$ such that $\bar{d}(t)=N / \psi$. We then show that 
given the properties of utility and of the production functions for leisure and work, the departure rate must be smaller as initial departure time becomes larger, which enables us to locate a single departure time that meets both the total population condition and the Nash equilibrium condition.

We begin by defining abstract functions $\rho, R$, and $u$ whose properties we will gradually restrict so that they can represent the departure rate, cumulative departures, and utility for a Nash equilibrium.

Definition 1 An anchored function $R(d \mid t)$ is a real function defined for $d \geq 0$ and $t \in[0, \Omega]$, with $R(0 \mid t)=0$. It is associated with a utility profile defined as

$u(d \mid t)=U\left(t+\int_{0}^{d} g_{H}\left(1-\frac{R(s \mid t)}{N}\right) d s, \Omega-\left(t+\frac{N}{\psi}\right)+\frac{1}{\psi} \int_{R(d \mid t)}^{N} g_{W}\left(\frac{s}{N}\right) d s\right)$.

Note that the utility profile depends only on $R(\cdot)$ and on the utility and agglomeration functions. The term "anchored" means simply that it is required to start at zero, i.e., time $t$ represents an initial departure.

Definition 2 A candidate cumulative departure function is an anchored function $R(\cdot \mid t)$ whose utility profile is constant:

$$
0=\frac{\partial u(d \mid t)}{\partial d}=U_{H} \cdot g_{H}\left(1-\frac{R(d \mid t)}{N}\right)-U_{W} \cdot g_{W}\left(\frac{R(d \mid t)}{N}\right) \frac{\rho(d \mid t)}{\psi}
$$

where

$$
\rho(d \mid t)=\frac{\partial R(d \mid t)}{\partial d}
$$

is called the candidate departure rate profile.

Remark 1 Equation (23) and the properties of $U, g_{H}$, and $g_{W}$, along with the anchoring requirement that $R(0 \mid t)=0$, imply that $\rho(\cdot \mid t)>0$ and

$$
\rho(d \mid t) \underset{d \rightarrow 0^{+}}{\longrightarrow} \infty
$$

Definition 3 Given a candidate departure rate profile, a candidate departure du- 
ration $\bar{d}(t)$ is defined implicitly by the total population condition

$$
\int_{0}^{\bar{d}(t)} \rho(s \mid t) d s=N
$$

By continuity, Lemma 3 below ensures that there is at least one $t$ with $\bar{d}(t)=$ $N / \psi$. Since $\rho(\cdot \mid t)$ is decreasing by Lemma $4, R(\cdot \mid t)$ is concave. Hence $R(d \mid t) \geq$ $\psi d$ for all $d \in] 0, N / \psi[$. Then there will be queue from time $t$ to time $t+N / \psi$ and so $u(d \mid t)$ describes the evolution of utility under the departure schedule $R(\cdot \mid t)$, which then describes a Nash equilibrium. Thus existence of Nash equilibrium is established.

It remains to show that Nash equilibrium is unique. Consider two Nash equilibria indexed by $a$ and $b$, starting departures at time $t_{a}$ and $t_{b}$, respectively, where $t_{a}<t_{b}$. Consider then the inequalities

$$
H_{a}\left(R^{-1}\left(x \mid t_{a}\right)\right)<H_{b}\left(R^{-1}\left(x \mid t_{b}\right)\right), W_{a}\left(R^{-1}\left(x \mid t_{a}\right)\right)>W_{b}\left(R^{-1}\left(x \mid t_{b}\right)\right) .
$$

Then (24) is valid at $x=0$. It follows by continuity that there exists $x^{\prime}>0$ such that (24) is valid for all $x<x^{\prime}$. Then by Condition 2,

$$
\rho\left(R^{-1}\left(x \mid t_{a}\right) \mid t_{a}\right)>\rho\left(R^{-1}\left(x \mid t_{b}\right) \mid t_{b}\right)
$$

for all such $x$. This implies that $R^{-1}\left(x \mid t_{a}\right)<R^{-1}\left(x \mid t_{b}\right)$ and so (24) holds also at $x=x^{\prime}$. This argument shows that (24) holds at all $x \in[0,1]$, since there can be no first $x$ where it fails. But this is a contradiction since

$$
\frac{N}{\psi}=\int_{0}^{N} \frac{1}{\rho\left(R^{-1}\left(x \mid t_{a}\right) \mid t_{a}\right)} d x<\int_{0}^{N} \frac{1}{\rho\left(R^{-1}\left(x \mid t_{b}\right) \mid t_{b}\right)} d x=\frac{N}{\psi} .
$$

This establishes that Nash equilibrium is unique.

Lemma 3 Conditions 1 and 2 imply that a candidate departure duration brackets the value $N / \psi$, as follows:

$$
\bar{d}(0)<\frac{N}{\psi}<\bar{d}\left(\Omega-\frac{N}{\psi}\right)
$$


Proof. Consider the first inequality and assume on the contrary that $\bar{d}(0) \geq \frac{N}{\psi}$. Note that $\rho(0 \mid 0)=\infty$, which rules out that $R(d \mid 0)<\psi d$ for all $d>0$ by the definition of $\rho$ as the derivative of $R$. Then $\exists d>0$ s.t. $R(d \mid 0)=\psi d$. There is queue for all departures in $[0, d]$ and so utility is constant for departures in this interval. But $H=0$ for departure at time 0 and so utility is $-\infty$ at this departure time while utility is finite for departure at time $d$. This is a contradiction and we conclude that $\bar{d}(0)<\frac{N}{\psi}$. Consider now the second inequality and assume on the contrary that $\frac{N}{\psi} \geq \bar{d}\left(\Omega-\frac{N}{\psi}\right)$. This implies that there is queue for all departures in the interval $[\Omega-N / \psi, \Omega-N / \psi+\bar{d}(\Omega-N / \psi)]$ and hence utility is constant for departure times in this interval. For the last possible departure time we have $W=0$ and hence $U=-\infty$, while $U$ is finite for the first departure time. This is a contradiction and we conclude that $\frac{N}{\psi}<\bar{d}\left(\Omega-\frac{N}{\psi}\right)$ as desired.

Lemma 4 Consider Nash equilibrium with first departure at time $t_{0}$. Then $\rho^{\prime}\left(d \mid t_{0}\right)<$ 0 for $d \in \operatorname{int}(I)$.

Proof. The departure rate $\rho\left(d \mid t_{0}\right)$ satisfies

$$
0=U_{H} \cdot g_{H}\left(1-\frac{R\left(d \mid t_{0}\right)}{N}\right)-U_{W} \cdot g_{W}\left(\frac{R\left(d \mid t_{0}\right)}{N}\right) \frac{\rho\left(d \mid t_{0}\right)}{\psi} .
$$

Differentiate and rearrange slightly to find that

$$
\begin{aligned}
& U_{W} \cdot g_{W}\left(\frac{R\left(d \mid t_{0}\right)}{N}\right) \frac{\rho^{\prime}\left(d \mid t_{0}\right)}{\psi} \\
= & \left(U_{H H} H^{\prime}+U_{H W} W^{\prime}\right) \cdot H^{\prime}+\left(U_{H W} H^{\prime}+U_{W W} W^{\prime}\right) \cdot W^{\prime} \\
& -U_{H} \cdot g_{H}^{\prime}\left(1-\frac{R\left(d \mid t_{0}\right)}{N}\right) \frac{\rho\left(d \mid t_{0}\right)}{N}-U_{W} \cdot g_{W}^{\prime}\left(\frac{R\left(d \mid t_{0}\right)}{N}\right) \frac{\rho\left(d \mid t_{0}\right)^{2}}{N \psi} .
\end{aligned}
$$

The RHS of this equation is strictly negative since $U$ is strictly concave and $U_{H}>$ $0, U_{W}>0$. The desired conclusion follows.

Proof of Theorem 2. (a) Nash equilibrium requires equal utilities, since all workers are identical, and thus in particular it requires (7). The proofs of existence 
and uniqueness are similar to those for our model of endogenous scheduling, and also to those for the bottleneck model, so are omitted here.

(b) First we differentiate equation (7), using (5) for $t_{1}$, to obtain:

$$
\tilde{V}^{\prime}\left(t_{0}\right) \frac{\partial t_{0}}{\partial \psi}=\tilde{V}^{\prime}\left(t_{1}\right)\left(\frac{\partial t_{0}}{\partial \psi}-\frac{N}{\psi^{2}}\right) .
$$

Solving,

$$
\frac{\partial t_{0}}{\partial \psi}=-\frac{N}{\psi^{2}} \frac{\widetilde{V}^{\prime}\left(t_{1}\right)}{\widetilde{V}^{\prime}\left(t_{0}\right)-\widetilde{V}^{\prime}\left(t_{1}\right)}>0 .
$$

Applying these results, we can differentiate (8) to obtain (9).

(c) The optimal toll eliminates congestion but never leaves the bottleneck delivering less than its full capacity until the end of the departure period. The logic here is the same as in Arnott et al. (1993): any congestion would cause a loss of scheduling utility without a compensating gain; but any unused capacity would permit reallocating someone to a departure time with a higher utility. That this toll is the one satisfying (10) can be seen by noting that with no queue, $\widetilde{V}(t)$ is the actual scheduling utility received by a traveler departing at $t$ (in contrast to the situation before tolling, where scheduling utility was lower because arrival $a$ differed from departure $t$ for all but the first and last traveler). This traveler's utility net of toll payment is the left-hand side of (10), which therefore states that this net utility is constant in time, making the new departure and arrival schedule a Nash equilibrium. In the case $\widetilde{\tau}\left(t_{0}\right)=0,(10)$ shows that this net utility is identical to that received before the toll was introduced.

(d) Total welfare change for a worker is defined as the change in that worker's net utility, plus toll revenues received from that worker. Each worker achieves the same utility net of toll payments as before the toll is imposed. Therefore, the net utility change to all workers is zero, and the total welfare change is equal to total revenues.

Proof of Theorem 3. For any $t, a \in\left(t_{0}, t_{1}\right)$, we have $0<1-R\left(t-t_{0} \mid t_{0}\right) / N<$ 1 and $0<\psi \cdot\left(a-t_{0}\right) / N<1$. We need to show that $V(t, a)$ is increasing in departure time $t$, decreasing in arrival time $a$, and strictly concave; and that $\widetilde{V}(t)=V(t, t)$ attains a maximum. The first two statements can be shown by 
signing the derivatives of $V(t, a)$ :

$$
\begin{aligned}
& V_{1}(t, a)=U_{H} \cdot g_{H}\left[1-\frac{R\left(t-t_{0} \mid t_{0}\right)}{N}\right]>0 \\
& V_{2}(t, a)=-U_{W} \cdot g_{W}\left[\frac{\psi \cdot\left(a-t_{0}\right)}{N}\right]<0,
\end{aligned}
$$

where $U_{H}$ and $U_{W}$ are evaluated at $H(t)$ and $W(a)$. To establish concavity, we compute the second derivatives of $V$ :

$$
\begin{aligned}
& V_{11}(t, a)=U_{H H} \cdot g_{H}^{2}-U_{H} \cdot g_{H}^{\prime} \frac{\rho\left(t-t_{0} \mid t_{0}\right)}{N}<0 \\
& V_{22}(t, a)=U_{W W} \cdot g_{W}^{2}-U_{W} \cdot g_{W}^{\prime} \frac{\psi}{N}<0 \\
& V_{12}(t, a)=-U_{H W} \cdot g_{H} \cdot g_{W}<0,
\end{aligned}
$$

where the arguments of $g_{H}$ and $g_{W}$ are the same as in (25). Strict concavity of $V$ is equivalent to its Hessian being negative definite. That is, the following quadratic form must be strictly negative for any real numbers $x_{1}, x_{2} \neq 0$ :

$$
\begin{aligned}
& x_{1}^{2} \cdot V_{11}(t, a)+2 x_{1} x_{2} \cdot V_{12}(t, a)+x_{2}^{2} \cdot V_{22}(t, a) \\
= & x_{1}^{2} \cdot U_{H H} \cdot g_{H}^{2}-x_{1}^{2} \cdot U_{H} \cdot g_{H}^{\prime} \cdot \frac{\rho\left(t-t_{0} \mid t_{0}\right)}{N} \\
& -2 x_{1} x_{2} \cdot U_{H W} \cdot g_{H} \cdot g_{W} \\
& +x_{2}^{2} \cdot U_{W W} \cdot g_{W}^{2}-x_{2}^{2} \cdot U_{W} \cdot g_{W}^{\prime} \cdot \frac{\psi}{N} \\
< & \left(x_{1} \cdot g_{H}\right)^{2} \cdot U_{H H} \\
& -2\left(x_{1} \cdot g_{H}\right) \cdot\left(x_{2} \cdot g_{W}\right) \cdot U_{H W} \\
& +\left(x_{2} \cdot g_{W}\right)^{2} \cdot U_{W W} .
\end{aligned}
$$

This expression is indeed strictly negative because $U$ is strictly concave.

It remains to show that $\widetilde{V}(t)=V(t, t)$ attains a maximum. This follows since $\widetilde{V}$ inherits concavity from $V$ and since $\widetilde{V}\left(t_{0}\right)=\widetilde{V}\left(t_{1}\right)$.

Proof of Theorem 4. A Vickrey-like analyst would set a toll schedule $\widetilde{\tau}(t)$ aimed at maintaining a departure schedule starting at $t_{0}$ which just eliminates queueing: namely $\widetilde{R}\left(s \mid t_{0}\right)=\psi s$. He does so by calculating this schedule based on the 
assumed scheduling utility function $V(t, a)$ given by (12) with $a=t$ (de Palma and Fosgerau, 2011). This utility can be written as follows:

$$
\widetilde{V}(t) \equiv V(t, t)=U\left[t_{0}+\int_{0}^{t-t_{0}} g_{H}\left(1-\frac{R\left(s \mid t_{0}\right)}{N}\right) d s, \widehat{W}\left(t \mid t_{0}\right)\right]
$$

where

$$
\widehat{W}\left(t \mid t_{0}\right)=\int_{t-t_{0}}^{t_{1}-t_{0}} g_{W}\left(\frac{\psi s}{N}\right) d s+\Omega-\left(t_{0}+N / \psi\right) .
$$

and $R(\cdot)$ follows its no-toll equilibrium path as depicted in Fig. 1. We can assume the arbitrary toll constant would be chosen so that $\widetilde{\tau}\left(t_{0}\right)=0$. The utility toll would thus be:

$$
\widetilde{\tau}(t)=\widetilde{V}(t)-\widetilde{V}\left(t_{0}\right)
$$

since this would assure that the anticipated utility net of toll would be identical for everyone under the desired departure pattern. This desired pattern, which we denote by $\widetilde{R}(\cdot)$, is the same as the line labeled $N_{W}$ in Figure 1 .

As an intermediate step toward a truly optimal toll, consider now an analyst who also aims to achieve departure pattern $\widetilde{R}(\cdot)$, but who knows the true model. This analyst would similarly use (27) except with $\widetilde{V}(t)$ replaced by the true utility given that departure pattern. That utility accounts for how $H$ changes as a result of the change in departure pattern: it is calculated as in (26) but with new cumulative departures $\psi s$ replacing the original cumulative departures $R(s \mid t)$ in the argument of $g_{H}$. Denoting the result by $\hat{V}(t)$, we have:

$$
\hat{V}(t) \equiv U\left[t_{0}+\int_{0}^{t-t_{0}} g_{H}\left(1-\frac{\psi s}{N}\right) d s, \widehat{W}\left(t \mid t_{0}\right)\right]
$$

The utility toll $\widehat{\tau}(t)$ would thus be set to maintain utility net of toll at its original constant value $\widetilde{\Delta}=\widetilde{V}\left(t_{0}\right)$, i.e. it is given by (14). Because $\psi s<R\left(s \mid t_{0}\right)$ for every value of $s$ in the arguments of $g_{H}$, the value of $H$ (i.e., the first argument of $U[\cdot]$ in these equations) is greater in (28) than in (26). Therefore $\hat{V}(t)>\widetilde{V}(t)$ and as a consequence $\hat{\tau}(t)>\widetilde{\tau}(t)$, for every $t>t_{0}$.

Now consider the welfare gains from toll $\hat{\tau}(t)$. A generalized Vickrey analyst would believe the welfare gain to be equal to the toll revenue from $\widetilde{\tau}(\cdot)$, 
with constant departure rate $\rho=\psi$ : that is, $\psi \int_{t_{0}}^{t_{1}} \widetilde{\tau}(t) d t$. But we have just seen that the congestion-removing toll that really leaves workers' net utility unaffected, namely $\hat{\tau}(t)$, is larger than $\widetilde{\tau}(t)$, and strictly larger for $t>t_{0}$. The departure rate with that toll is again $\rho=\psi$, so its welfare gain (the sum of all travelers' utilities) is equal to toll revenue $\psi \int_{t_{0}}^{t_{1}} \hat{\tau}(t) d t$. Hence the toll revenue and welfare gain are both strictly larger than believed by Vickrey.

Proof of Lemma 2. (a and b) From the definition of $\widetilde{V}$ and eq. (12), we have $\widetilde{V}^{\prime}\left(t_{0}\right)=d V\left(t_{0}, t_{0}\right) / d t_{0}=V_{1}\left(t_{0}, t_{0}\right)+V_{2}\left(t_{0}, t_{0}\right)=U_{H}^{0} \cdot g_{H}(1)-U_{W}^{0} \cdot g_{W}(0)$ $=U_{H}^{0}$. Similarly, $\widetilde{V}^{\prime}\left(t_{1}\right)=V_{1}\left(t_{1}, t_{1}\right)+V_{2}\left(t_{1}, t_{1}\right)=U_{H}^{1} \cdot g_{H}(0)-U_{W}^{1} \cdot g_{W}(1)$ $=-U_{W}^{1}$. Then use Theorem 2. (c) Equation (17) is obtained by differentiating equilibrium utility $U^{0}$ in (15) with respect to $\psi$.

\section{B Numerical simulation details}

\section{B.1 Nash equilibrium}

For given parameters $\psi, \alpha, \pi_{W}$, and $\pi_{H}$, the numerical solution proceeds by first choosing a trial value of $t_{0}$ and dividing the time axis into many small periods starting at $t_{0}$. We then compute $\rho$ and $R$ stepwise. For the first few periods, cumulative departures are computed from (31) as explained below; after that they are computed by cumulating values of departure rate $\rho$, which is computed in each time interval from (21) using values of $R, H$, and $W$ as determined in the previous period. When $R$ is near one, we apply (32) to find the end of the departure interval. We compare the duration of this departure interval with its required value, $N / \psi$, to determine whether the trial value of $t_{0}$ is consistent with equilibrium; if not, we adjust $t_{0}$ iteratively until the correct interval length is achieved. This is an instance of the "shooting method".

Finally, we check the accuracy by comparing the values of $U$ computed at all the time periods; they should be identical, and if not we make the time steps smaller in order to increase the accuracy of the calculation. We typically get satisfactory results with 500 time periods, achieving identical utilities to within about 0.1 percent, and within about 0.03 percent for all but the first 10 time periods; 
we have also verified that the solution remains stable for a range of grid sizes approaching the one we use.

The procedure for values of $t$ near $t_{0}$ or $t_{1}$ is different because equation (21) is numerically unsatisfactory there. First, the departure rate $\rho$ is infinite at $R=0$, the beginning of the rush hour. Furthermore, as $R$ approaches 1 (the end of the departure interval), $\rho \rightarrow 0$, causing that approach to possibly occur very slowly since $R$ is the integral of $\rho$. These extremes make a simple stepwise numerical procedure inaccurate, so instead we calculate $R$ in those two regions by solving an approximate version of (21) for constant $U_{H} / U_{W}$. This is quite accurate because $H$ and $W$ contain constants at the boundaries, which allows the marginal utilities $\alpha / H$ and $1 / W$ to be nearly constant there. Furthermore, near the first boundary we can approximate $[1-R(\cdot)]$ in (21) as a constant equal to unity, and near the second boundary we can approximate $R(\cdot)$ as a constant equal to unity. The approximate differential equations are then

$$
\begin{array}{lr}
\rho=K_{0} \cdot R^{-\pi_{W}} & \text { near } t=t_{0}, \\
\rho=K_{1} \cdot(1-R)^{\pi_{H}} & \text { near } t=t_{1},
\end{array}
$$

where $K_{0}=\psi U_{H}(0) / U_{W}(0)$ and $K_{1}=\psi U_{H}(1) / U_{W}(1)$ with notation $(0)$ and (1) indicating values at the endpoints of the departure interval. We seek the solutions to these differential equations, with boundary conditions $R\left(0 \mid t_{0}\right)=0$ for (29) and $R\left(\frac{N}{\psi} \mid t_{0}\right)=1$ for (30). The solutions are:

$$
\begin{array}{ll}
R=\left[\left(1+\pi_{W}\right) \cdot K_{0} \cdot\left(t-t_{0}\right)\right]^{\frac{1}{\pi_{w}+1}} & \text { near } t=t_{0}, \\
1-R=\left[\left(t_{1}-t\right)\left(1-\pi_{H}\right) K_{1}\right]^{\frac{1}{1-\pi_{h}}} \quad \text { near } t=t_{1} .
\end{array}
$$

Note that (32) requires $\pi_{H}<1$ to be valid, i.e. to give $(1-R)$ as a finite real number. Basically, this is because for larger values of $\pi_{H}$, the departure rate (30) becomes so small as $R \rightarrow 1$ that the limiting value $R=1$, indicating the end of the departure period, can never be reached. 


\section{B.2 Optimum}

The maximization in (22) is performed by writing out the arguments of $U[\widehat{H}(t), \widehat{W}(t)]$ in the integrand as functions of $t_{d}=t-t_{0}$ :

$$
\begin{aligned}
\widehat{H}\left(t_{d} \mid t_{0}\right) & =t_{0}+\int_{0}^{t_{d}}(1-\psi s)^{\pi_{H}} d s=t_{0}+\frac{1}{\psi} \cdot \frac{1-\left(1-\psi t_{d}\right)^{\pi_{H}+1}}{\pi_{H}+1} \\
W\left(t_{d} \mid t_{0}\right) & =\Omega-t_{1}+\frac{1}{\psi} \cdot \int_{t_{d}}^{1 / \psi}(\psi s)^{\pi_{W}} d s=\Omega-t_{0}-\frac{1}{\psi}+\frac{1-\left(\psi t_{d}\right)^{\pi_{W}+1}}{\pi_{W}+1}
\end{aligned}
$$

where now we have explicitly indicated in the notation that these arguments depend on $t_{0}$. (There is not carat on $W$ when written as a function of $t_{d}$ because, conditional on $t_{0}$, it is the same function as in the Nash equilibrium calculation.) The average value of this utility is

$$
\bar{U}\left(t_{0}\right)=\psi \int_{0}^{1 / \psi} U\left[\widehat{H}\left(t_{d} \mid t_{0}\right), W\left(t_{d} \mid t_{0}\right)\right] d t_{d}
$$

and the first-order condition for maximizing it is

$0=\frac{d \bar{U}}{d t_{0}}=\psi \int_{0}^{1 / \psi} U_{H} \frac{\partial \widehat{H}}{\partial t_{0}} d t_{d}+\psi \int_{0}^{1 / \psi} U_{W} \frac{\partial W}{\partial t_{0}} d t_{d}=\psi \int_{0}^{1 / \psi} \frac{\alpha}{\widehat{H}} d t_{d}-\psi \int_{0}^{1 / \psi} \frac{1}{W} d t_{d}$

since $\partial \widehat{H} / \partial t_{0}=1$ and $\partial W / \partial t_{0}=-1$. Intuitively, (33) states that $t_{0}$ is increased until the marginal benefit of increased home time, measured by $U_{H}$, is just matched by the marginal disbenefit of decreased work time, $U_{W}$. The internal dynamics, i.e. the shape of the departure pattern within the congested period, play no role in this calculation because they are unaffected by changes in $t_{0}$ given that the toll has been adjusted to make this departure pattern simply a constant at rate $\psi$.

\section{B.3 Capacity expansion}

The values of capacity expansion are given by (16) and (17), which here take the forms: 


$$
\begin{gathered}
\frac{\partial \widetilde{\Delta}}{\partial \psi}=\frac{N}{\psi^{2}} \frac{\alpha}{H^{0}+\alpha W^{1}} \\
\frac{\partial \Delta}{\partial \psi}=\left[\frac{\alpha}{t_{0}}-\frac{1}{W^{0}}\right] \frac{\partial t_{0}}{\partial \psi}+\frac{1}{W^{0}} \frac{N}{\psi^{2}} \frac{\pi_{W}}{1+\pi_{W}}
\end{gathered}
$$

where $H^{0}$ and $W^{0}$ are the values taken by $H$ and $W$ for the first traveler, and $W^{1}$ is the value taken by $W$ for the last traveler.

\section{B.4 Practical issues}

The simulations are actually performed with time measured in units of 2 hours. Parameters and results with a time dimension are rescaled accordingly in presenting results: specifically, $\Omega$ and $N / \Psi$ are simulated with values equal to one-half the stated values, and all resulting times are inflated by a factor of 2 . In addition, the time universe $[0, \Omega]$ is taken to represent the interval [2:00 a.m., 12:00 noon] in simulations \#1-5, and [midnight, noon] in simulation \#6; hence 2 hours are added to clock time $t_{0}$ when reporting results in simulations \#1-5. 